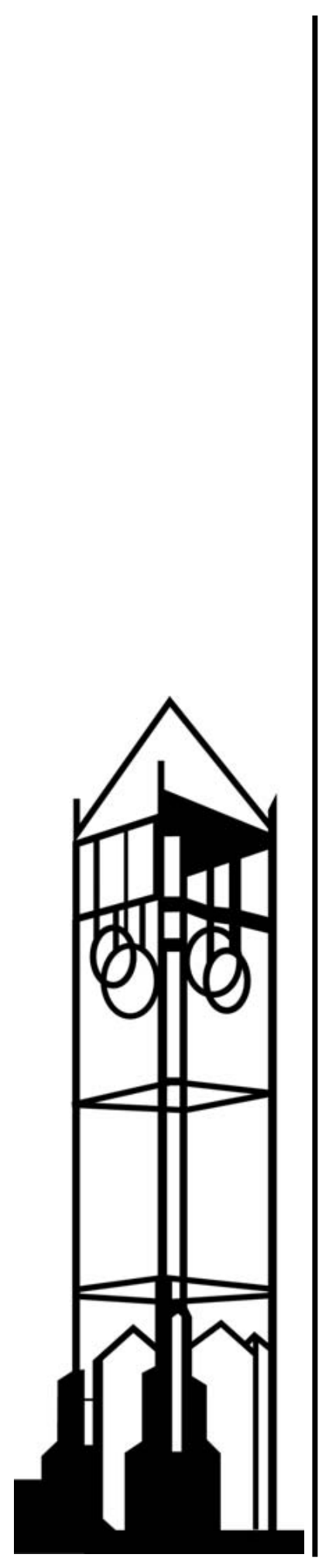

Food Stamps and Food Insecurity: What Can Be Learned in the Presence of Non-Classical Measurement Error?

Craig Gundersen, Brent Kreider

Working Paper No. 06034

IOWA STATE UNIVERSITY

Department of Economics

Ames, lowa, 50011-1070

lowa State University does not discriminate on the basis of race, color, age, religion, national origin, sexual orientation, gender identity,sex, marital status, disability, or status as a U.S. veteran. Inquiries can be directed to the Director of Equal Opportunity and Diversity, 3680 Beardshear Hall, (515) 294-7612. 


\section{IOWA STATE UNIVERSITY}

\begin{tabular}{|c||}
\hline $\begin{array}{c}\text { Food Stamps and Food Insecurity: What Can Be } \\
\text { Learned in the Presence of Non-classical Measurement } \\
\text { Error? }\end{array}$ \\
Craig Gundersen, Brent Kreider \\
October 2006 \\
Working Paper \# 06034 \\
Department of Economics \\
Working Papers Series
\end{tabular}

Ames, Iowa 50011

Iowa State University does not discriminate on the basis of race, color, age, religion, national origin, sexual orientation, gender identity, sex, marital status, disability, or status as a U.S. veteran. Inquiries can be directed to the Director of Equal Opportunity and Diversity, 3680 Beardshear Hall, (515) $294-7612$. 


\title{
Food Stamps and Food Insecurity: What Can Be Learned in the Presence of Nonclassical Measurement Error?*
}

\author{
Craig Gundersen \\ Iowa State University \\ cggunder@iastate.edu
}

\author{
Brent Kreider ${ }^{\dagger}$ \\ Iowa State University \\ bkreider@iastate.edu
}

March 2007

\begin{abstract}
Policymakers have been puzzled to observe that food stamp households appear more likely to be food insecure than observationally similar eligible nonparticipating households. We reexamine this issue allowing for nonclassical reporting errors in food stamp participation and food insecurity. Extending the literature on partially identified parameters, we introduce a nonparametric framework that makes transparent what can be known about conditional probabilities when a binary outcome and conditioning variable are both subject to nonclassical measurement error. We find that the food insecurity paradox hinges on strong assumptions about the reliability of the data that are not supported by the previous food stamp participation literature.
\end{abstract}

Keywords: Food Stamp Program, food insecurity, measurement error, nonparametric bounds, corrupt sampling

JEL classification: $\mathrm{H} 5, \mathrm{I} 32, \mathrm{I} 12$

${ }^{*}$ This research is funded through a grant from the U.S. Department of Agriculture (USDA), Economic Research Service and the Institute for Research on Poverty. The views expressed in this article are solely those of the authors. Previous versions of this paper were presented at the Annual Meetings of the Association for Public Policy Analysis and Management (APPAM), the ERS/IRP Small Grants Research Workshop, the Annual Meetings of the Population Association of America, the Annual Meetings of the American Council on Consumer Interests, and the Research Innovation and Development Grants in Economics (RIDGE) Conference. The authors thank participants at those venues and, in particular, Karl Scholz, Mark Nord, Hugette Sun, and Sandi Hoffereth.

${ }^{\dagger}$ Corresponding author: Department of Economics, Iowa State University, Ames, IA 50011, tel: (515) 294-6237, fax: (515) 294-0221, email: bkreider@iastate.edu. 


\section{Introduction}

The largest food assistance program in the United States, the Food Stamp Program is "... the most critical component of the safety net against hunger (U.S. Department of Agriculture, 1999, p.7)." While this program provides basic protection for citizens of all ages and household status, the safety net is especially important for children who comprise over half of all recipients (Cunnyngham and Brown, 2004). Given the cornerstone role of food stamps in ensuring food security, policymakers have been puzzled to observe that food stamp households appear more likely to be food insecure than observationally similar nonparticipating eligible households.

In response to a burgeoning interest in food insecurity, an extensive literature has developed in the last decade on the determinants and consequences of food insecurity in the United States. ${ }^{1}$ The negative association between food security and food stamp participation has been ascribed to several factors including self-selection based on unobserved household characteristics, the timing of food insecurity versus food stamp receipt, misreporting of food insecurity status, and misreporting of food stamp receipt. Previous work has studied these first two issues (e.g., Gundersen and Oliveira, 2001; Wilde and Nord, 2005; Nord et al., 2004). The literature has not assessed the consequences of measurement error.

We focus on measurement error issues using data from the Core Food Security Module (CFSM), a component of the Current Population Survey (CPS). Specifically, we investigate what can be inferred when food stamp participation and food insecurity status may be misreported. As elaborated below, we extend the econometric literature on misclassified binary variables by studying identification when an outcome (in our case food insecurity) and a conditioning variable (food stamp participation) are both subject to arbitrary endogenous classification error. We also consider the identifying power of assumptions that restrict the patterns of classification errors. For example, misreported food insecurity status might arise independently of true food stamp participation status. We propose a computationally efficient estimation algorithm for this "orthogonal errors" case. We also consider cases involving asymmetric reporting errors. As is well understood in the econometrics literature, even random errors can lead to seriously biased parameter estimates.

A number of studies have documented the presence of substantial reporting error in households' receipt of

\footnotetext{
${ }^{1}$ For other recent work see, e.g., Bhattacharya et al., 2004; Bitler et al., 2005; Borjas, 2004; Dunifon and KowaleskiJones, 2003; Furness et al., 2004; Gundersen et al., 2003; Laraia et al., 2006; Ribar and Hamrick, 2003; and Van Hook and Balistreri, 2006.
} 
food stamp benefits. For example, using administrative data matched with data from the Survey of Income and Program Participation (SIPP), Marquis and Moore (1990) found that about 19 percent of actual food stamp recipient households reported that they were not recipients. Underreporting of up to 25 percent has also been documented in comparisons between responses in surveys, such as the CPS, and administrative data (Cunnyngham, 2005). Bollinger and David (1997, 2001, 2005) estimate econometric models of food stamp response errors and study the consequences of misreporting for inference on take-up rates.

The assumption of fully accurate reporting of food insecurity status can also be questioned. Even in anonymous surveys, some food stamp recipients might misreport being food insecure if they believe that to report otherwise could jeopardize their eligibility. ${ }^{2}$ Alternatively, some parents might misreport being food secure if they feel ashamed about heading a household in which their children are not getting enough food to eat (Hamelin et al., 2002). More generally, some of the survey questions used to calculate official food insecurity status (see Section 2) require the respondent to make a subjective judgment. Validation studies consistently reveal large degrees of response error in survey data for a wide range of self-reports, even for relatively objective variables (see, e.g., Black et al., 2003). Moreover, timing issues in the comparison of food insecurity status against the receipt of food stamps can further confound identification.

In this paper, we study what can be known about food insecurity status conditional on food stamp participation when both variables may be misreported. The econometric component of the paper extends the literature on misclassified binary variables (e.g., Aigner, 1973; Bollinger, 1996; Bollinger and David, 1997, 2001; Frazis and Loewenstein, 2003; Kreider and Pepper, forthcoming $a, b$ ). Our nonparametric framework follows the spirit of Horowitz and Manski (1995) who study partial identification under corrupt samples given minimal assumptions on the error generating process. ${ }^{3}$ Within this environment, we derive sharp worst-case bounds on conditional food insecurity rates that exploit all available information under the maintained assumptions.

To isolate the identification problem associated with potentially misreported food stamp participation, we begin by assuming that the food insecurity outcome is reported without error. First, we present sharp bounds derived in Kreider and Pepper (forthcoming, a) that impose no assumptions on the patterns of

\footnotetext{
${ }^{2}$ Other literatures contain lively debates about the extent to which self-reported disability might be influenced by a respondent's desire to rationalize labor force withdrawal or the receipt of disability benefits (see, e.g., Bound and Burkhauser, 1999).

${ }^{3}$ For extensions of their nonparametric approach, see, for example, Hotz et al. (1997), Pepper (2000), Dominitz and Sherman (2004), Molinari (2005), and Kreider and Pepper (forthcoming a).
} 
classification errors in a conditioning variable. Next, we derive narrower sets of bounds for two benchmark cases that impose structure on the reporting error process. In Proposition 1, we consider the identifying power of an assumption that food stamp participation is potentially underreported but households do not falsely claim to receive food stamps. In Proposition 2, we introduce easy-to-compute sharp bounds for the case that food stamp misreporting arises independently of true participation status (orthogonal errors). For these bounds, we show how to transform a computationally expensive multidimensional search problem into a series of single-dimension search problems that requires little programming effort or computational time. After studying the identification problem for the case of fully accurate food insecurity responses, we consider the case that food insecurity as well as food stamp participation may be reported with error. ${ }^{4}$

In the next section, we describe the central variables of interest in this paper - food insecurity and food stamps - followed by a description of the CFSM data. In Section 3, we highlight the statistical identification problem created by the potential unreliability of the self-reported data. We then show how conditional food insecurity prevalence rates can be partially identified under various restrictions on the nature and degree of classification errors. Section 4 presents our empirical results, and Section 5 concludes.

\section{Concepts and Data}

\subsection{Food Insecurity}

The extent of food insecurity in the United States has become a well-publicized issue of concern to policymakers and program administrators. In 2003, $11.2 \%$ of the U.S. population reported that they suffered from food insecurity at some time during the previous year. As described below, these households were uncertain of having, or unable to acquire, enough food for all their members because they had insufficient money or other resources. For about $3.5 \%$ of the population, the degree of food insecurity was severe enough to be recorded as food insecurity with hunger. For households with children, the reported levels were higher: $16.7 \%$ and $3.8 \%$, respectively.

To calculate the official food insecurity rates in the U.S., defined over a 12 month period, a series of 18 questions are posed in the CSFM for families with children. ${ }^{5}$ Each question is designed to capture some aspect of food insecurity and, for some questions, the frequency with which it manifests itself. Examples include "I

\footnotetext{
${ }^{4}$ In contrast to our nonparametric approach, Hausman et al. (1998) propose parametric and semiparametric estimators in a discrete-response regression setting that allow for misclassification in a dependent variable.

${ }^{5}$ For families without children and for one-person households, a subset of 10 questions are posed.
} 
worried whether our food would run out before we got money to buy more" (the least severe outcome); "Did you or the other adults in your household ever cut the size of your meals or skip meals because there wasn't enough money for food?" and "Did a child in the household ever not eat for a full day because you couldn't afford enough food?" A household with children is categorized as (a) food secure if the respondent responds affirmatively to two or fewer of these questions, (b) food insecure if the respondent responds affirmatively to three or more questions, and (c) food insecure with hunger if the respondent responds affirmatively to eight or more questions. ${ }^{6}$ A complete listing of the food insecurity questions is presented in Table 1 . Figure 1 provides the frequency of affirmative responses to each of the 18 CFSM questions for each food insecurity category (food secure, food insecure without hunger, and food insecure with hunger).

The CFSM questions are designed to portray food insecurity in the United States in a manner consistent with how experts perceive the presence of food insecurity. Given conceptual difficulties in quantifying food insecurity status, its measurement contains both objective and subjective components. ${ }^{7}$ Such classifications are thus akin to classifications of work disability in that work capacity involves both objective factors (e.g., the presence of a medical condition) and subjective factors (e.g., the ability to function effectively despite the presence of the condition). ${ }^{8}$ For reasons described above, a household's food insecurity status might be misclassified relative to the profession's intended threshold for true food insecurity.

The ordered nature of some of the CFSM food insecurity questions provides a limited check on the consistency of the responses. For example, "Child skipped meal" (item \#16) is arguably more severe than "Child was hungry" (\#14), which in turn is arguably more severe than "Child not eating enough" (\#9). Moreover, "Child skipped meal(s) for three or more months (\#17)" and "Child did not eat for whole day" (\#18) are more severe than the preceding items, though \#17 and \#18 are not ordered themselves given a potential tradeoff between intensity and duration of hunger. Pervasive inconsistencies in responses to ordered questions would presumably heighten concerns about the reliability of the household food insecurity indicators.

\footnotetext{
${ }^{6}$ In 1996, the label "food insecurity with hunger" was changed to "very low food security status." Despite this change in nomenclature, the methods used to define households are identical under the two names. We treat food insecurity as a binary indicator in this paper consistent with how it is generally defined by researchers and policymakers. We do not attempt to address conceptual issues about how food insecurity should be ideally quantified.

${ }^{7}$ Consistent with the subjective nature of the questions in the CFSM, Gundersen and Ribar (2005) find that selfreported food insecurity has a substantially higher correlation with a subjective measure of food expenditure needs than with an objective measure of such needs.

${ }^{8}$ See, for example, Bound and Burkhauser (1999).
} 
To investigate inconsistency rates, we propose the following sets of orderings: $\{9,14,16,17\}$ and $\{9,14,16,18\}$ (child skipped meals), $\{5,8,15\}$ and $\{5,13,15\}$ (adults skipped meals), and $\{7,10,11\}$ (respondent did not eat enough). There might be additional reasonable orderings, but we conservatively focus on the most obvious cases. Based on these sets, we find at least one inconsistency for $6.1 \%$ of the sample. Most of these inconsistencies are concentrated on questions pertaining to adult members of the household; for the questions about children, the inconsistency rate is only about 1\%. Of course, the presence (or absence) of such inconsistencies does not by itself confer knowledge about the reliability of a household's aggregate food insecurity classification. Food insecurity can be misclassified even if the household always reports consistently, and the presence of inconsistencies is not necessarily pivotal in determining the aggregate outcome. Still, we have reason to question the reliability of $6.1 \%$ of the responses even before considering the other aforementioned sources of potential classification error.

\subsection{The Food Stamp Program}

The Food Stamp Program, with a few exceptions, is available to all families with children who meet income and asset tests. To receive food stamps, households must meet three financial criteria: a gross-income test, a net-income test, and an asset test. A household's gross income before taxes in the previous month cannot exceed 130 percent of the poverty line, and net monthly income cannot exceed the poverty line. ${ }^{9}$ Finally, income-eligible households with assets less than $\$ 2,000$ qualify for the program. The value of a vehicle above $\$ 4,650$ is considered an asset unless it is used for work or for the transportation of disabled persons. Households receiving Temporary Assistance for Needy Families (TANF) and households where all members receive Supplemental Security Income (SSI) are categorically eligible for food stamps and do not have to meet these three tests.

A large fraction of households eligible for food stamps do not participate. This outcome is often ascribed to three main factors. First, there may be stigma associated with receiving food stamps. Stigma encompasses a wide variety of sources, from a person's own distaste for receiving food stamps to the fear of disapproval from others when redeeming food stamps to the possible negative reaction of caseworkers (Ranney and

\footnotetext{
${ }^{9}$ Net income is calculated by subtracting a standard deduction from a household's gross income. In addition to this standard deduction, households with labor earnings deduct 20 percent of those earnings from their gross income. Deductions are also taken for child care and/or care for disabled dependents, medical expenses, and excessive shelter expenses.
} 
Kushman, 1987; Moffitt, 1983). Second, transaction costs can diminish the attractiveness of participation. ${ }^{10}$ A household faces these costs on a repeated basis when it must recertify its eligibility. Third, against these costs, the benefit level may be too small to induce participation; food stamp benefits can be as low as $\$ 10$ a month for a family.

Reported food stamp participation in survey data may deviate from actual participation. Evidence of this underreporting has surfaced in two types of studies, both of which compare self-reported information with official records. The first type has compared aggregate statistics obtained from self-reported survey data with those obtained from administrative data. These studies suggest the presence of substantial underreporting of food stamp recipiency. In the CFSM data used in our analysis, Bitler et al. (2003, Table 3) find that the number of food stamp recipients in the 1999 CFSM reflected only about 85 percent of the true number according to administrative data. Similar undercounts have been observed in the March Supplement of the CPS, the Survey of Income and Program Participation (SIPP), the Panel Study of Income Dynamics (PSID), and the Consumer Expenditure Survey (CES) (Trippe et al., 1992). Other studies have compared individual reports of food stamp participation status in surveys with matched reports from administrative data. Using this method, researchers can identify both errors of commission (i.e., reporting benefits not actually received) and errors of omission (i.e., not reporting benefits actually received). Using data from the SIPP, Bollinger and David (1997, Table 2) find that, consistent with aggregate reports, 0.3 percent of households have errors of commission while 12.0 percent have errors of omission.

\subsection{Data}

Our analysis uses data from the December Supplement of the 2003 CPS. The CPS is the official data source for poverty and unemployment rates in the U.S. and has included the CFSM component at least one month in every year since 1995. In 2003, this component was included in the December Supplement. The December CPS also contains information on food stamp participation status. For our main analysis, we define households as self-reported food stamp participants if they report the receipt of food stamps at some point over the previous 12 months. Similarly, the standard CFSM questions measure the occurrence of food insecurity over the previous 12 months. ${ }^{11}$ We limit our sample to households with children eligible for the

\footnotetext{
${ }^{10}$ Examples of such costs include travel time to a food stamp office and time spent in the office, the burden of transporting children to the office or paying for child care services, and the direct costs of paying for transportation.

${ }^{11}$ Ideally, there should be a close correspondence between the time frames that the households report their food insecurity status and food stamp receipt. We specifically choose the 12 month time horizons, however, to be consistent
} 
Food Stamp Program based on the gross income criterion. Our sample of 2707 observations consists of all households with children reporting incomes less than 130 percent of the poverty line. ${ }^{12}$

Table 2 displays joint frequency distributions of reported food insecurity status and food stamp participation for our sample of eligible households with children. Panel A shows that 52.3\% (582/1113) of households who reported the receipt of food stamps also reported being food insecure. Among households who did not report the receipt of food stamps, 34.4\% (549/1594) reported being food insecure. Based on these responses, the prevalence of food insecurity is 17.9 percentage points higher among food stamp recipients than among eligible nonrecipients. Based on analogous information in Panel B, the prevalence of food insecurity with hunger is 6.5 percentage points higher among food stamp recipients (15.9\%) than among eligible nonrecipients $(9.4 \%)$. In what follows, we assess what can be inferred about these conditional prevalence rates when food stamp participation and food insecurity status are subject to classification errors.

\section{Identification}

To assess the impact of classification error on inferences, we introduce notation that distinguishes between reported food stamp participation status and true participation status. Let $X^{*}=1$ indicate that a household truly receives food stamps, with $X^{*}=0$ otherwise. Instead of observing $X^{*}$, we observe a self-reported counterpart $X$. A latent variable $Z^{*}$ indicates whether a report is accurate: $Z^{*}=1$ if $X^{*}=X$, with $Z^{*}=0$ otherwise. Finally, let $Y=1$ denote that a household reports being food insecure, with $Y=0$ otherwise. Initially, we focus exclusively on food stamp misclassifications and assume that food insecurity status is measured without error. We later allow for the possibility of misclassifications in both food stamp participation and food insecurity status.

Taking self-reports at face value, we can point-identify the food insecurity prevalence rates among food stamp recipients and nonrecipients as 0.523 and 0.344 , respectively (Table $2 \mathrm{~A}$ ) - a difference that is statistically significant at better than the $1 \%$ level. Allowing for the possibility of classification errors, however, we

with previous studies that have investigated relationships between these two variables (e.g., Wilde and Nord, 2005). In Section 4.3, we consider alternative definitions.

${ }^{12}$ Our data do not contain sufficient information for us to apply the net income test or asset test. However, virtually all families meeting the gross income test also meet the net income test. The asset test could be important for a sample that includes a high proportion of households headed by an elderly person (Haider et al., 2003). For households with children, however, the fraction asset ineligible but gross income eligible is small. Using combined data from 1989 to 2004 in the March CPS (which does have information on the returns to assets), Gundersen and Offutt (2005) find that only $7.1 \%$ of households with children are asset ineligible but gross income eligible. 
cannot identify $P\left(Y=1 \mid X^{*}\right)$ even if reporting errors are thought to occur randomly. To formalize the identification problem, consider the rate of food insecurity among the true population of food stamp recipients. This conditional probability is given by

$$
P\left(Y=1 \mid X^{*}=1\right)=\frac{P\left(Y=1, X^{*}=1\right)}{P\left(X^{*}=1\right)} .
$$

Since one does not observe $X^{*}$, neither the numerator nor the denominator is identified. ${ }^{13}$ However, assumptions on the pattern of reporting errors can place restrictions on relationships between the unobserved quantities. Let $\theta_{1}^{+} \equiv P\left(Y=1, X=1, Z^{*}=0\right)$ and $\theta_{1}^{-} \equiv P\left(Y=1, X=0, Z^{*}=0\right)$ denote the fraction of false positive and false negative food stamp participation classifications, respectively, within the population of food-insecure households. Similarly, let $\theta_{0}^{+} \equiv P\left(Y=0, X=1, Z^{*}=0\right)$ and $\theta_{0}^{-} \equiv P\left(Y=0, X=0, Z^{*}=0\right)$ denote the fraction of false positive and false negative food stamp participation classifications, respectively, within the population of food-secure households. Then we can decompose the numerator and denominator in (1) into identified and unidentified quantities:

$$
P\left(Y=1 \mid X^{*}=1\right)=\frac{p_{11}+\theta_{1}^{-}-\theta_{1}^{+}}{p+\left(\theta_{1}^{-}+\theta_{0}^{-}\right)-\left(\theta_{1}^{+}+\theta_{0}^{+}\right)}
$$

where $p_{11} \equiv P(Y=1, X=1)$ and $p \equiv P(X=1)$ are identified by the data and the other quantities are not identified. In the numerator, $\theta_{1}^{-}-\theta_{1}^{+}$reflects the unobserved excess of false negative vs. false positive food stamp participation reports for the population of food-insecure households. In the denominator, $\left(\theta_{1}^{-}+\theta_{0}^{-}\right)-\left(\theta_{1}^{+}+\theta_{0}^{+}\right)$reflects the excess of false positive vs. false negative classifications within the entire population of interest. The food insecurity prevalence rate among nonrecipients can be written analogously as

$$
P\left(Y=1 \mid X^{*}=0\right)=\frac{p_{10}+\theta_{1}^{+}-\theta_{1}^{-}}{1-p+\left(\theta_{1}^{+}+\theta_{0}^{+}\right)-\left(\theta_{1}^{-}+\theta_{0}^{-}\right)}
$$

where $p_{10} \equiv P(Y=1, X=0)$.

Worst-case bounds on $P\left(Y=1 \mid X^{*}\right)$ are obtained by finding the extrema of equations (2) and (3) subject to restrictions on the false positives and false negatives $\theta_{1}^{+}, \theta_{0}^{+}, \theta_{1}^{-}$, and $\theta_{0}^{-}$. Without assumptions on the

\footnotetext{
${ }^{13}$ For ease of exposition, our notation leaves implicit any other conditioning variables (e.g., variables pertaining to household composition).
} 
nature of reporting errors, the following constraints hold:

$$
\begin{gathered}
\text { (i) } 0 \leq \theta_{1}^{+} \leq P(Y=1, X=1) \equiv p_{11} \\
\text { (ii) } 0 \leq \theta_{0}^{+} \leq P(Y=0, X=1) \equiv p_{01} \\
\text { (iii) } 0 \leq \theta_{1}^{-} \leq P(Y=1, X=0) \equiv p_{10} \\
\text { (iv) } 0 \leq \theta_{0}^{-} \leq P(Y=0, X=0) \equiv p_{00} .
\end{gathered}
$$

For example, the fraction of food insecure households that falsely reports receiving food stamps obviously cannot exceed the fraction of food insecure households that reports receiving food stamps.

Before considering any structure on the pattern of false positives and false negatives, we begin by assessing identification given a limit on the potential degree of misclassification. Following Horowitz and Manski (1995) and the literature on robust statistics (e.g., Huber 1981), we can study how identification of an unknown parameter varies with the confidence in the data. Consider an upper bound, $q$, on the fraction of inaccurate food stamp participation classifications: $P\left(Z^{*}=0\right) \leq q$ which implies

$$
\text { (v) } \theta_{1}^{+}+\theta_{0}^{+}+\theta_{1}^{-}+\theta_{0}^{-} \leq q \text {. }
$$

This assumption incorporates a researcher's beliefs about the potential degree of data corruption. If $q$ equals 0 (as is implicitly assumed in all previous work on food insecurity), then $P\left(Y=1 \mid X^{*}\right)$ is pointidentified because all food stamp participation reports are assumed to be accurate. At the opposite extreme, a researcher unwilling to place any limit on the potential degree of reporting error can set $q$ equal to 1 . In that case, there is no hope of learning anything about $P\left(Y=1 \mid X^{*}\right)$ without constraining the pattern of reporting errors. In any event, the sensitivity of inferences on $P\left(Y=1 \mid X^{*}\right)$ can be examined by varying the value of $q$ between 0 and 1 .

In the "corrupt sampling" case in which nothing is known about the pattern of reporting errors, we compute sharp bounds on $P\left(Y=1 \mid X^{*}\right)$ using a result from Kreider and Pepper (forthcoming, $a$ ). After briefly presenting these bounds, we derive narrower sets of bounds by imposing structure on the reporting error process. We first consider the identifying power of an assumption that respondents may fail to report receiving food stamps but not falsely claim to receive food stamps. We then consider the case that food stamp misreporting arises independently of true participation status. After establishing sets of bounds on $P\left(Y=1 \mid X^{*}\right)$ for the case that food insecurity is accurately reported, we allow for the possibility that food 
insecurity status may also be misreported. Throughout this analysis, we do not impose the nondifferential errors assumption embedded in the classical errors-in-variables framework. ${ }^{14}$

\subsection{Corrupt sampling bounds}

Under arbitrary errors (corrupt sampling), the researcher makes no assumptions about the patterns of false positive and false negative classifications. We can compute closed-form sharp "degree" bounds in this environment using the following result:

"Corrupt Sampling Degree Bounds" (Kreider-Pepper, forthcoming a, Prop. 1): Let $P\left(Z^{*}=0\right) \leq q$, and suppose that food insecurity is measured without error. Then the prevalence of food insecurity among food stamp participants is bounded sharply as follows:

$$
\frac{p_{11}-\alpha^{+}}{p-2 \alpha^{+}+q} \leq P\left(Y=1 \mid X^{*}=1\right) \leq \frac{p_{11}+\alpha^{-}}{p+2 \alpha^{-}-q}
$$

using the values

$$
\begin{aligned}
& \alpha^{+}= \begin{cases}\min \left\{q, p_{11}\right\} & \text { if } p_{11}-p_{01}-q \leq 0 \\
\max \left\{0, q-p_{00}\right\} & \text { otherwise }\end{cases} \\
& \alpha^{-}= \begin{cases}\min \left\{q, p_{10}\right\} & \text { if } p_{11}-p_{01}+q \leq 0 \\
\max \left\{0, q-p_{01}\right\} & \text { otherwise. }\end{cases}
\end{aligned}
$$

Analogous bounds for the prevalence of food insecurity among nonrecipients, $P\left(Y=1 \mid X^{*}=0\right)$, are obtained by replacing $X=1$ with $X=0$ and vice versa in each of the relevant quantities.

Naturally, these bounds can be narrowed if the researcher is willing to make assumptions that restrict the pattern of reporting errors. Suppose, for example, that the researcher believes that food stamp participation is potentially underreported but households do not falsely claim to receive food stamps. This assumption is consistent with the evidence discussed above regarding errors of omission and errors of commission (Bollinger and David, 2001). In this case, we can simply impose $\theta_{1}^{+}=\theta_{0}^{+}=0$ in Equations (2) and (3). The sharp lower bound on $P\left(Y=1 \mid X^{*}=1\right)$ is attained when $\theta_{1}^{-}=0$ and $\theta_{0}^{-}=\min \left\{q, p_{00}\right\}$ while the sharp upper bound is attained when $\theta_{0}^{-}=0$ and $\theta_{1}^{-}=\min \left\{q, p_{10}\right\}$. Similarly, the sharp lower bound on $P\left(Y=1 \mid X^{*}=0\right)$ is attained when $\theta_{0}^{-}=0$ and $\theta_{1}^{-}=\min \left\{q, p_{10}\right\}$ while the sharp upper bound is attained when $\theta_{1}^{-}=0$ and

\footnotetext{
${ }^{14}$ In our context, this assumption would require that, conditional on true participation status, participation classification errors arise independently of food insecurity status. Bollinger (1996) studies identification of a mean regression when a potentially mismeasured binary conditioning variable satisfies the nondifferential errors assumption.
} 
$\theta_{0}^{-}=\min \left\{q, p_{00}\right\}$. As a straightforward extension of the corrupt sampling bounds, we obtain the closed-form "no false positives" bounds:

Proposition 1: Let $P\left(Z^{*}=0\right) \leq q$ and suppose that households do not falsely report the receipt of food stamps. Suppose further that food insecurity is measured without error. Then the conditional food insecurity prevalence rates are bounded sharply as follows:

$$
\begin{gathered}
\frac{p_{11}}{p+\min \left\{q, p_{00}\right\}} \leq P\left(Y=1 \mid X^{*}=1\right) \leq \frac{p_{11}+\min \left\{q, p_{10}\right\}}{p+\min \left\{q, p_{10}\right\}} \\
\frac{p_{10}-\min \left\{q, p_{10}\right\}}{1-p-\min \left\{q, p_{10}\right\}} \leq P\left(Y=1 \mid X^{*}=0\right) \leq \frac{p_{10}}{1-p-\min \left\{q, p_{00}\right\}}
\end{gathered}
$$

The assumption of no false positive reports does not always improve all of the bounds. For example, suppose that the allowed degree of classification error is small enough that $q \leq \min \left\{p_{00}, p_{11}-p_{01}\right\}$. In this case, the lower bound on $P\left(Y=1 \mid X^{*}=1\right)$ under the assumption of no false positive reports is identical to the lower bound under corrupt sampling.

\subsection{Orthogonal participation errors}

Many studies have assumed that classification errors arise independently of the variable's true value (see Molinari (2005) for a discussion). Bollinger (1996), for example, discusses the possibility that a worker's true union status has no influence on whether union status is misreported in the data. Kreider and Pepper (forthcoming, $b$ ) consider the identifying assumption that, among certain types of respondents, misreported disability status does not depend on true disability status. ${ }^{15}$ In the present context, this independence assumption implies that misreporting of food stamp participation is orthogonal to true participation status:

$$
P\left(X^{*}=1 \mid Z^{*}=1\right)=P\left(X^{*}=1 \mid Z^{*}=0\right) .
$$

In this case, the false positive and false negative classification errors must satisfy the constraint:

$$
\begin{array}{r}
(v i) \quad\left[1-p-\left(\theta_{1}^{-}+\theta_{0}^{-}\right)\right]\left[\left(\theta_{1}^{+}+\theta_{0}^{+}\right)+\left(\theta_{1}^{-}+\theta_{0}^{-}\right)\right] \\
=\left(\theta_{1}^{+}+\theta_{0}^{+}\right)\left[1-\left(\theta_{1}^{+}+\theta_{0}^{+}\right)-\left(\theta_{1}^{-}+\theta_{0}^{-}\right)\right] .
\end{array}
$$

\footnotetext{
${ }^{15}$ In contrast to our analysis, their objective is to place bounds on an unknown marginal distribution. They do not consider misclassification of a conditioning variable.
} 
Based on earlier discussion, there is reason to believe that food stamp reporting errors are not random. Nevertheless, the orthogonality assumption is weaker than the usual assumption of no classification errors, and it serves as a useful benchmark case for comparison.

Sharp bounds on the conditional food insecurity rates, $P\left(Y=1 \mid X^{*}\right)$, can be found by searching over all feasible combinations of $\left\{\theta_{1}^{+}, \theta_{0}^{+}, \theta_{1}^{-}, \theta_{0}^{-}\right\}$in (2) subject to satisfying constraint (vi). Computational costs associated with a simultaneous search over three of these four parameters (after imposing the constraint), however, can quickly become burdensome at high values of $q$ - especially while bootstrapping to obtain confidence intervals or when combining the independence assumption with other restrictions. ${ }^{16}$ As we elaborate below, our Proposition 2 provides simple-to-compute bounds that require only single-dimension searches.

We focus discussion on deriving a lower bound on $P\left(Y=1 \mid X^{*}=1\right)$. Differentiating (2), it can be shown that $P\left(Y=1 \mid X^{*}=1\right)$ is increasing in $\theta_{1}^{-}$and $\theta_{0}^{+}$and decreasing in $\theta_{0}^{-}$and $\theta_{1}^{+}$. Given independence, however, we cannot rule out the possibility that the lower bound involves positive values of $\theta_{1}^{-}$or $\theta_{0}^{+}$. Increasing these values above zero allows for the possibility of increasing $\theta_{0}^{-}$or $\theta_{1}^{+}$while remaining on the independence contour.

To obtain a computationally expedient lower bound on $P\left(Y=1 \mid X^{*}=1\right)$ given orthogonal errors, we analyze a series of exhaustive cases. The smallest calculated lower bound across these cases establishes the lower bound of interest. We proceed using the following outline: In Case 1, we derive the lower bound under the possibility that $\theta_{1}^{-}=\theta_{0}^{+}=0$. In Case 2 , we derive the lower bound under the possibility that $\theta_{1}^{-}=0$ and $\theta_{0}^{+}>0$. In this case, the lower bound cannot involve $\theta_{0}^{+}>0$ unless $\theta_{1}^{+}=p_{11}$ which eliminates $\theta_{1}^{+}$as an unknown parameter. Cases 3 and 4 are similar. The lower bound on $P\left(Y=1 \mid X^{*}=1\right)$ is then obtained as the smallest lower bound derived under four possible cases. In each case, there exists only one free parameter to search across after imposing the independence constraint.

Case 1: $\theta_{1}^{-}=\theta_{0}^{+}=0$ :

When $\theta_{1}^{-}=\theta_{0}^{+}=0$, there are two free parameters in Equation (2): $\theta_{1}^{+}$and $\theta_{0}^{-}$. For any candidate value of $\theta_{1}^{+}$, the independence constraint $(v i)$ constrains $\theta_{0}^{-}$to be one of two values: $\theta_{0, j}^{-}\left(\theta_{1}^{+}\right) \equiv$ $\frac{(1-p)+(-1)^{j} \sqrt{(1-p)^{2}-4 \theta_{1}^{+}\left(p-\theta_{1}^{+}\right)}}{2}$ for $j=1,2$. By constraint $(i)$, the fraction of respondents who reported being

\footnotetext{
${ }^{16}$ For example, one might impose a monotone instrumental variables (MIV) assumption (Manski and Pepper, 2000) that true food insecurity varies monotonically with particular variables. In this case, the cells would need to be further partitioned for each allowed value of the instrumental variable.
} 
food insecure and misreported being a food stamp participant cannot exceed the fraction who participated and reported being food insecure: $\theta_{1}^{+} \in\left[0, \overline{\theta_{1}^{+}}\right]$. By constraint $(i v)$, the fraction of respondents that did not participate and misreported being food secure cannot exceed the fraction that did not participate in the Food Stamp Program and reported being food secure: $\theta_{0}^{-} \in\left[0, \overline{\theta_{0}^{-}}\right]$. By constraint $(v)$, the total fraction of misreporters $\theta_{1}^{+}+\theta_{0}^{-}$cannot exceed $q$. Therefore, the lower bound when $\theta_{1}^{-}=\theta_{0}^{+}=0$ is given by

$$
L B_{1}=\inf _{\theta_{1}^{+} \in \Theta_{j}^{1}, j=1,2} \frac{p_{11}-\theta_{1}^{+}}{p+\theta_{0, j}^{-}\left(\theta_{1}^{+}\right)-\theta_{1}^{+}}
$$

where $\Theta_{j}^{1} \equiv\left[0, \overline{\theta_{1}^{+}}\right] \cap\left\{\theta_{1}^{+}: \theta_{0, j}^{-}\left(\theta_{1}^{+}\right) \in\left[0, \overline{\theta_{0}^{-}}\right]\right\} \cap\left\{\theta_{1}^{+}: \theta_{1}^{+}+\theta_{0, j}^{-}\left(\theta_{1}^{+}\right) \leq q\right\}$ and $\theta_{0, j}^{-}\left(\theta_{1}^{+}\right) \equiv \frac{(1-p)+(-1)^{j} \sqrt{(1-p)^{2}-4 \theta_{1}^{+}\left(p-\theta_{1}^{+}\right)}}{2}$ for $j=1,2$.

From a practical standpoint, this lower bound is obtained by simply searching for the smallest value of $\frac{p_{11}-\theta_{1}^{+}}{p+\theta_{0, j}^{-}\left(\theta_{1}^{+}\right)-\theta_{1}^{+}}$across feasible values of $\theta_{1}^{+} \in\left[0, \overline{\theta_{1}^{+}}\right]$. Feasible values of $\theta_{1}^{+}$include those associated with a value of $\theta_{0, j}^{-}$that lies in the allowed range $\left[0, \overline{\theta_{0}^{-}}\right]$, subject to the requirement that the $\operatorname{sum} \theta_{1}^{+}+\theta_{0, j}^{-}$is not too large.

Case 2: $\theta_{1}^{-}=0, \theta_{0}^{+}>0$ :

First notice that $\theta_{1}^{+}$and $\theta_{0}^{+}$are perfectly substitutable in constraints $(v)$ and $(v i)$. Moreover, differentiating (2) when $\theta_{1}^{-}=0$ reveals that increasing $\theta_{1}^{+}$lowers the ratio in (2) by more than raising the value of $\theta_{0}^{+}$(for any values of $\theta_{1}^{+}, \theta_{0}^{+}$, and $\theta_{0}^{-}$). Therefore, the optimal value of $\theta_{0}^{+}$cannot exceed zero unless $\theta_{1}^{+}$has attained its maximum feasible value $\overline{\theta_{1}^{+}}$. The lower bound when $\theta_{1}^{-}=0$ and $\theta_{0}^{+}>0$ is given by

$$
L B_{2}=\inf _{\theta_{0}^{-} \in \Theta_{j}^{2}, j=1,2} \frac{p_{11}-\overline{\theta_{1}^{+}}}{p+\theta_{0}^{-}-\overline{\theta_{1}^{+}}-\theta_{0, j}^{+}\left(\theta_{0}^{-}\right)}
$$

where $\Theta_{j}^{2} \equiv\left[0, \overline{\theta_{0}^{-}}\right] \cap\left\{\theta_{0}^{-}: \theta_{0, j}^{+}\left(\theta_{0}^{-}\right) \in\left(0, \overline{\theta_{0}^{+}}\right]\right\} \cap\left\{\theta_{0}^{-}: \overline{\theta_{1}^{+}}+\theta_{0, j}^{+}\left(\theta_{0}^{-}\right)+\theta_{0}^{-} \leq q\right\}$ and $\theta_{0, j}^{+}\left(\theta_{0}^{-}\right) \equiv \frac{p-2 \overline{\theta_{1}^{+}}+(-1)^{j} \sqrt{p^{2}-4 \theta_{0}^{-}\left(1-p-\theta_{0}^{-}\right)}}{2}$ for $j=1,2$.

Case 3: $\theta_{0}^{+}=0, \theta_{1}^{-}>0$ :

Similar to Case $2, \theta_{1}^{-}$and $\theta_{0}^{-}$are perfectly substitutable in constraints $(v)$ and $(v i)$. Differentiating (2) when $\theta_{0}^{+}=0$ reveals that increasing $\theta_{0}^{-}$lowers the ratio by more than raising the value of $\theta_{1}^{-}$(for any values of $\theta_{1}^{+}, \theta_{1}^{-}$, and $\left.\theta_{0}^{-}\right)$. Therefore, the optimal value of $\theta_{1}^{-}$cannot exceed zero unless $\theta_{0}^{-}$has attained its 
maximum feasible value $\overline{\theta_{0}^{-}}$. The lower bound when $\theta_{0}^{+}=0$ and $\theta_{1}^{-}>0$ is given by

$$
L B_{3}=\inf _{\theta_{1}^{-} \in \Theta_{j}^{3}, j=1,2} \frac{p_{11}+\theta_{1}^{-}-\theta_{1 j}^{+}\left(\theta_{1}^{-}\right)}{p+\theta_{1}^{-}+\overline{\theta_{0}^{-}}-\theta_{1 j}^{+}\left(\theta_{1}^{-}\right)}
$$

where $\Theta_{j}^{3} \equiv\left(0, \overline{\theta_{1}^{-}}\right] \cap\left\{\theta_{1}^{-}: \theta_{1 j}^{+}\left(\theta_{1}^{-}\right) \in\left[0, \overline{\theta_{1}^{+}}\right]\right\} \cap\left\{\theta_{1}^{-}: \overline{\theta_{0}^{-}}+\theta_{1 j}^{+}\left(\theta_{1}^{-}\right)+\theta_{1}^{-} \leq q\right\}$

and $\theta_{1 j}^{+}\left(\theta_{1}^{-}\right) \equiv \frac{p+(-1)^{j} \sqrt{p^{2}-4\left(\overline{\theta_{0}^{-}}+\theta_{1}^{-}\right)\left(1-p-\theta_{1}^{-}-\overline{\theta_{0}^{-}}\right)}}{2}$ for $j=1,2$.

Case 4: $\theta_{1}^{-}>0, \theta_{0}^{+}>0$ :

Given $\theta_{1}^{+}=\overline{\theta_{1}^{+}}$and $\theta_{0}^{-}=\overline{\theta_{0}^{-}}$when $\theta_{1}^{-}$and $\theta_{0}^{+}$are positive, the lower bound when $\theta_{1}^{-}>0$ and $\theta_{0}^{+}>0$ is given by

$$
L B_{4}=\inf _{\theta_{0}^{+} \in \Theta_{j}^{4}, j=1,2} \frac{p_{11}+\theta_{1 j}^{-}\left(\theta_{0}^{+}\right)-\overline{\theta_{1}^{+}}}{p+\theta_{1 j}^{-}\left(\theta_{0}^{+}\right)+\overline{\theta_{0}^{-}}-\overline{\theta_{1}^{+}}-\theta_{0}^{+}}
$$

where $\Theta_{j}^{4} \equiv\left(0, \overline{\theta_{0}^{+}}\right] \cap\left\{\theta_{0}^{+}: \theta_{1 j}^{-}\left(\theta_{0}^{+}\right) \in\left(0, \overline{\theta_{1}^{-}}\right]\right\} \cap\left\{\theta_{0}^{+}: \overline{\theta_{1}^{+}}+\overline{\theta_{0}^{-}}+\theta_{1 j}^{-}\left(\theta_{0}^{+}\right)+\theta_{0}^{+} \leq q\right\}$

and $\theta_{1 j}^{-}\left(\theta_{0}^{+}\right) \equiv \frac{1-p-2 \overline{\theta_{0}^{-}}+(-1)^{j} \sqrt{(1-p)^{2}-4\left(\overline{\theta_{1}^{+}}+\theta_{0}^{+}\right)\left(p-\overline{\theta_{1}^{+}}-\theta_{0}^{+}\right)}}{2}$ for $j=1,2$.

Combining these results with analogous results for upper bounds, we obtain the following proposition:

Proposition 2. Let $P\left(Z^{*}=0\right) \leq q$, and suppose that food insecurity is measured without error. Then sharp bounds on $P\left(Y=1 \mid X^{*}=1\right)$ under the orthogonal errors assumption in (4) are identified as

$$
\Omega_{L} \leq P\left(Y=1 \mid X^{*}=1\right) \leq \Omega_{H}
$$

where $\Omega_{L} \equiv \inf \left\{L B_{1}, L B_{2}, L B_{3}, L B_{4}\right\}$ and $\Omega_{H} \equiv \sup \left\{U B_{1}, U B_{2}, U B_{3}, U B_{4}\right\}$. Analogous bounds on $P(Y=$ $\left.1 \mid X^{*}=0\right)$ are obtained by replacing $X=1$ with $X=0$, and vice versa, in the relevant quantities.

The expressions for the upper bounds are provided in Appendix A. ${ }^{17}$ The bounds converge to the selfreported conditional food insecurity rate $P(Y=1 \mid X=1)$ as $q$ goes to 0 . Increasing $q$ may widen the bounds over some ranges of $q$ but not others, and the rate of identification decay can be highly nonlinear as $q$ increases.

\footnotetext{
${ }^{17}$ For sufficiently high values of $q$, some values lying between the worst-case lower and upper bounds may not be feasible under the independence constraint of Equation (4); sharp identification regions can be constructed, if desired, by simply excluding such values.
} 
These bounds are easy to program, and computing time is trivial given that searches are conducted in a single dimension. To compute $L B_{1}$, for example, we need only to search over feasible values of $\theta_{1}^{+}$. In our application, computational speed for the Proposition 2 bounds at $q=0.5$ is more than 3300 times faster than the speed associated with a simultaneous search across three of the four parameters $\theta_{1}^{+}, \theta_{0}^{+}, \theta_{1}^{-}$, and $\theta_{0}^{-}$(reduced to three dimensions after incorporating the independence constraint). ${ }^{18}$ Moreover, the singledimensional search allows us to avoid specifying an arbitrary tolerance threshold for when independence is satisfied. If the specified tolerance is too small, the calculated bounds become artificially narrow as feasible bounds are excluded from consideration. In contrast, a large tolerance leads to unnecessarily conservative estimated bounds. In practice, we found it quite time-consuming to find a reasonable balance between speed and accuracy - a trade-off that varies across different values of $q$. The proposed single-dimension search procedure effectively avoids this problem.

\subsection{Food insecurity classification errors}

To this point, we have confined our attention to classification errors in food stamp participation. For reasons noted above, however, we might also suspect the presence of errors in food insecurity reports. Suppose that true food insecurity status is measured by the latent indicator $Y^{*}$. The observed indicator $Y$ matches the true value $Y^{*}$ if $Z^{* \prime}=1$ and is misclassified if $Z^{* \prime}=0$. Analogous to the case of misreported food stamp participation, let $q^{\prime}$ represent an upper bound on the allowed degree of corruption in $Y: P\left(Z^{* \prime}=\right.$ $0) \leq q^{\prime}$. Modifying Equation (1), the true food insecurity prevalence rate among food stamp recipients and nonrecipients is given by

$$
p_{1 k}^{*}=\frac{P\left(Y^{*}=1, X^{*}=k\right)}{P\left(X^{*}=k\right)} .
$$

where $p_{1 k}^{*} \equiv P\left(Y^{*}=1 \mid X^{*}=k\right)$ for $k=1,0$.

Given the possibility of classification errors in both $X$ and $Y$, there are now many more types of error combinations. We represent these combinations by $\theta_{j k}^{u v}$. The subscripts $j$ and $k$ indicate true food insecurity status and true food stamp participation status, respectively. Specifically, $j=1$ indicates that the household is truly food secure ( $j=0$ otherwise), and $k=1$ indicates that the household truly receives food stamps ( $k=0$ otherwise). The superscripts indicate whether these outcomes are falsely classified, and if so, in

\footnotetext{
${ }^{18}$ For different empirical applications, these values will vary depending on the quantities $p_{11}, p_{01}, p_{10}, p_{00}$ defined above.
} 
which direction. Specifically, $u=$ "+" indicates that the household is misclassified as food insecure, $u="-"$ indicates that the household is misclassified as food secure, and $u=$ " $o$ " indicates that food insecurity status is not misclassified. Similarly, $v="+"$ indicates that the household is misclassified as receiving benefits, $v=$ "-" indicates that the household is misclassified as not receiving benefits, and $v=$ " $o$ " indicates that participation status is not misclassified.

As before, we can decompose the numerator and denominator into observed and unobserved components:

$$
p_{11}^{*}=\frac{P(Y=1, X=1)+\left(\theta_{11}^{-o}+\theta_{11}^{o-}+\theta_{11}^{--}\right)-\left(\theta_{10}^{o+}+\theta_{01}^{+o}+\theta_{00}^{++}\right)}{P(X=1)+\left(\theta_{11}^{o-}+\theta_{11}^{--}+\theta_{01}^{+-}+\theta_{01}^{o-}\right)-\left(\theta_{10}^{o+}+\theta_{00}^{++}+\theta_{10}^{-+}+\theta_{00}^{o+}\right)} .
$$

Similarly, we can write

$$
p_{10}^{*}=\frac{P(Y=1, X=0)+\left(\theta_{10}^{-o}+\theta_{10}^{o+}+\theta_{10}^{-+}\right)-\left(\theta_{11}^{o-}+\theta_{00}^{+o}+\theta_{01}^{+-}\right)}{P(X=0)+\left(\theta_{10}^{o+}+\theta_{10}^{-+}+\theta_{00}^{++}+\theta_{00}^{o+}\right)-\left(\theta_{11}^{o-}+\theta_{01}^{+-}+\theta_{11}^{--}+\theta_{01}^{o-}\right)} .
$$

We can compute sharp bounds on $p_{11}^{*}$ and $p_{10}^{*}$ by searching across all feasible combinations of false positive and false negative classifications in $X^{*}$ and $Y^{*}$. The following constraints must hold, analogous to constraints $(i-i v)$ earlier:

$$
\begin{gathered}
\text { (i') } 0 \leq \theta_{01}^{+o}, \theta_{10}^{o+}, \theta_{00}^{++} \leq P(Y=1, X=1) \equiv p_{11} \\
\left(i i^{\prime}\right) \quad 0 \leq \theta_{00}^{o+}, \theta_{11}^{-o}, \theta_{10}^{-+} \leq P(Y=0, X=1) \equiv p_{01} \\
\left(i i i^{\prime}\right) \quad 0 \leq \theta_{00}^{+o}, \theta_{11}^{o-}, \theta_{01}^{+-} \leq P(Y=1, X=0) \equiv p_{10} \\
\left(\text { iv' }^{\prime}\right) \quad 0 \leq \theta_{10}^{-o}, \theta_{11}^{--}, \theta_{01}^{o-} \leq P(Y=0, X=0) \equiv p_{00} .
\end{gathered}
$$

For example, the fraction of households simultaneously misclassified as food insecure and misclassified as receiving food stamps, $\theta_{00}^{++}$, cannot exceed the fraction of households who report being food insecure with food stamps, $p_{11}$. The errors must also satisfy the constraints

$$
\left(v^{\prime}\right) \theta_{00}^{o+}+\theta_{10}^{-+}+\theta_{10}^{o+}+\theta_{00}^{++}+\theta_{11}^{o-}+\theta_{11}^{--}+\theta_{01}^{+-}+\theta_{01}^{o-} \leq q \text {. }
$$

and

$$
\left(v^{\prime \prime}\right) \theta_{11}^{-o}+\theta_{10}^{-+}+\theta_{01}^{+o}+\theta_{00}^{+o}+\theta_{00}^{++}+\theta_{01}^{+-}+\theta_{10}^{-o}+\theta_{11}^{--} \leq q^{\prime} \text {. }
$$

A search over all combinations of errors becomes rapidly burdensome as the values of $q$ and $q^{\prime}$ are allowed to rise. Nevertheless, the problem is feasible for sufficiently low degrees of potential data corruption. For 
the case of corrupt sampling, the search problem is greatly simplified because no structure is placed on the pattern of errors. In that case, many of the unknown parameters for each bound can be set to 0. For example, suppose we wish to compute a sharp lower bound on $p_{11}^{*}$. It is easy to see that the lower bound requires $\theta_{00}^{o+}=\theta_{10}^{-+}=\theta_{11}^{-o}=0$. Differentiation further reveals that $\theta_{11}^{--}=\theta_{11}^{o-}=0$ as well. Analogous restrictions arise for the other bounds. For the case that we assume orthogonal errors in $X$ and/or $Y$, we cannot set any of the parameters to 0 . Instead, we search over all feasible combinations of errors subject to the requirement that candidates for the bounds are discarded unless the appropriate orthogonality analogues to constraint $(v i)$ are satisfied..$^{19}$

We next turn to empirical results. We first illustrate what can be identified about conditional food insecurity prevalence rates under the assumption that the receipt of benefits may be misclassified but food insecurity is accurately measured. We then allow for the possibility that food insecurity is misreported as well. We pinpoint critical values of allowed degrees of data corruption for when we can no longer identify that food stamp recipients are more likely to be food insecure than eligible nonrecipients.

\section{Results}

\subsection{Food Stamp Classification Errors}

Figures 2 and 3 trace out patterns of identification decay for inferences on the prevalence of food insecurity among food stamp recipients and nonrecipients, respectively, as a function of the allowed degree of data corruption, $q$. As discussed above, we focus our attention on eligible households with children. For these figures, we assume that only food stamp participation is subject to classification error; food insecurity classifications are presumed to be accurate.

In Figure 2 we examine what can be known about $p_{11}^{*}$, the prevalence of food insecurity among food stamp recipients. When $q=0$, all food stamp classifications are taken at face value; uncertainty about the magnitude of $\Delta$ arises from sampling variability alone. As seen in the figure and the table beneath it, the prevalence rate at $q=0$ is point-identified as $p_{11}=0.523$ with $90 \%$ confidence interval $[0.496,0.545]$.

What can be known about $p_{11}^{*}$ when $q>0$ depends on what the researcher is willing to assume about the nature and degree of reporting errors. If $q=0.05$, then up to $5 \%$ of the food stamp classifications may be inaccurate. In this case under arbitrary errors, $p_{11}^{*}$ is partially identified to lie within the range $[0.457,0.595]$,

\footnotetext{
${ }^{19}$ Our Gauss computer code for computing these bounds is available upon request.
} 
a 14 point range. After accounting for sampling variability, this range expands to [0.427, 0.621], a 19 point range. The figure traces out the 5th percentile lower bound and 95th percentile upper bound across values of $q \cdot{ }^{20}$ The bounds naturally widen as our confidence in the reliability of the data declines. Once $q$ exceeds about 0.21 , we cannot say anything about the food insecurity rate of food stamp recipients; the prevalence rate could lie anywhere within $[0,1]$. The bounds narrow if we are willing to make assumptions about the pattern of errors. At $q=0.05$, for example, the bounds on $p_{11}^{*}$ narrow to $[0.461,0.586]$ under orthogonal errors (before accounting for sampling variability) and to $[0.466,0.575]$ under the no false positives assumption.

Figure 3 presents analogous bounds for $p_{10}^{*}$, the prevalence of food insecurity among nonrecipients. At $q=0$, this prevalence rate is point-identified as $p_{10}=0.344$, about 18 points lower than the food insecurity rate among recipients. For $q>0$, the orthogonality restriction substantially improves the lower bound relative to corrupt sampling. The upper bound, however, is not substantially improved except for high values of $q$. The assumption of no false positive classifications marginally improves the upper bound and has no effect on the lower bound.

Figure 4 provides sharp bounds on $\Delta \equiv p_{11}^{*}-p_{10}^{*}$, the difference in food insecurity rates (Figure $4 \mathrm{~A}$ ) and food insecurity with hunger rates (Figure 4B) between food stamp recipients and nonrecipients. A simple lower (upper) bound on $\Delta$ could be computed as the difference between the lower (upper) bound on $p_{11}^{*}$ and the upper (lower) bound on $p_{10}^{*}$. Such bounds would not be as tight as possible, however, because a different set of values of $\left\{\theta_{1}^{+}, \theta_{0}^{+}, \theta_{1}^{-}, \theta_{0}^{-}\right\}$might maximize (minimize) the expression in Equation (2) than would minimize (maximize) the expression in Equation (3). Instead, we obtain sharp bounds on $\Delta$ as follows:

$$
\begin{aligned}
& \Delta_{L B}=\min _{\theta_{1}^{+}, \theta_{0}^{+}, \theta_{1}^{-}, \theta_{0}^{-}}\left\{\frac{p_{11}+\theta_{1}^{-}-\theta_{1}^{+}}{p+\left(\theta_{1}^{-}+\theta_{0}^{-}\right)-\left(\theta_{1}^{+}+\theta_{0}^{+}\right)}-\frac{p_{10}+\theta_{1}^{+}-\theta_{1}^{-}}{1-p+\left(\theta_{1}^{+}+\theta_{0}^{+}\right)-\left(\theta_{1}^{-}+\theta_{0}^{-}\right)}\right\} \\
& \Delta_{U B}=\max _{\theta_{1}^{+}, \theta_{0}^{+}, \theta_{1}^{-}, \theta_{0}^{-}}\left\{\frac{p_{11}+\theta_{1}^{-}-\theta_{1}^{+}}{p+\left(\theta_{1}^{-}+\theta_{0}^{-}\right)-\left(\theta_{1}^{+}+\theta_{0}^{+}\right)}-\frac{p_{10}+\theta_{1}^{+}-\theta_{1}^{-}}{1-p+\left(\theta_{1}^{+}+\theta_{0}^{+}\right)-\left(\theta_{1}^{-}+\theta_{0}^{-}\right)}\right\}
\end{aligned}
$$

subject to all constraints imposed on the pattern of classification errors.

Figure 4A shows that small degrees of classification error are sufficient to overturn the conclusion from the data that $\Delta>0$, even without accounting for uncertainty arising from sampling variability. Under

\footnotetext{
${ }^{20}$ We bootstrap to obtain these values using the bias-corrected percentile method (Efron and Tibshirani, 1993) using 1,000 pseudosamples. The kinks at various values of $q$ reflect the impacts of constraints $(i)$ - $(v i)$ on allowed combinations of false positives and false negatives (Section 3$)$. For sufficiently small values of $q$, constraints $(i)$ - $(i v)$ are not binding because constraint $(v i)$ prevents $\theta_{1}^{+}, \theta_{0}^{+}, \theta_{1}^{-}$, or $\theta_{0}^{-}$from attaining their maximum feasible values. As $q$ rises, however, each of the other constraints eventually becomes binding, resulting in a kink in the figure. This kink is somewhat smoothed by bootstrapping across the pseudosamples.
} 
arbitrary errors, we cannot identify that $\Delta$ is positive if more than $7.1 \%$ of households might misreport their food stamp participation status. These critical values rise to $8.2 \%$ and $9.1 \%$ under orthogonal errors and no false positives, respectively. Panel B in the figure reproduces Panel A except that $Y^{*}=1$ is redefined as food insecurity with hunger. Here, we find that identification of the sign of $\Delta$ breaks down when $q$ is

only 0.018 under arbitrary errors and when $q$ is only 0.029 under orthogonal errors. Both are far lower than for the case of food insecurity. Under the assumption of no false positives, the critical value rises to 0.124. Again, these critical values are conservatively high in that they do not account for the additional uncertainty created by sampling variability.

As discussed in Section 2.2, Bollinger and David (1997) find that $12 \%$ of households fail to report their receipt of food stamps; evidence from Bitler et al. (2003) suggests the possibility of even greater degrees of undercounting. Thus, even before accounting for the possible mismeasurement of food insecurity status, we find it difficult to conclude that food insecurity is more prevalent among food stamp recipients than among eligible nonrecipients. Such a conclusion requires a large degree of confidence in self-reported food participation status. In the next section, we extend the analysis to the case that both food stamp recipiency and food insecurity may be misclassified. We then consider the sensitivity of our findings to alternative definitions and samples.

\subsection{Food stamp and food insecurity classification errors}

As discussed above, the possibility of food insecurity classification errors further confounds identification. Table 3 provides critical values for identification breakdown that vary across the different assumptions on the nature of classification errors. Row A reproduces information highlighted in Figure 4A for the case of fully accurate food insecurity classifications. Now suppose that food insecurity status might be misclassified for up to $5 \%$ of households: $q^{\prime}=0.05$. If these errors arise independently of true food insecurity status (Row B), then the sign of $\Delta$ cannot be identified under arbitrary program participation errors unless it is assumed that fewer than $2.8 \%$ of households might misreport their food stamp recipiency.

These critical values rise only slightly under the stronger assumptions of orthogonal food stamp errors (3.3\%) and no false positive food stamp reports (4.1\%). In Row C for the case of arbitrarily misreported food insecurity status, the critical values fall further to $2.1 \%, 2.4 \%$, and $3.5 \%$, respectively. Once $q^{\prime}$ exceeds 0.084 under orthogonal errors or 0.0073 under arbitrary errors, the sign of $\Delta$ is not identified even if all food 
stamp responses are known to be accurate. For the case of food insecurity with hunger, yet smaller degrees of uncertainty about the data are sufficient to lose identification of the sign of $\Delta$.

\subsection{Alternative time frames and samples}

We now examine the sensitivity of our main findings to alternative time frames and samples. We first consider the timing of food insecurity and food stamp receipt. Using the standard federal government classifications, our main analysis measures the occurrence of food insecurity and food stamp participation over the previous 12 months. Because these outcomes may change over the course of the year, however, this aggregation represents a potential source of measurement error distinct from misreporting. For example, some respondents might have been food insecure early in the year and then subsequently decided to receive food stamps. Even if food stamps alleviated the household's food insecurity, the household would be coded as a food insecure beneficiary. Thus, part of the observed positive relationship between food insecurity and program participation may be an artifact of mismatched time horizons.

To investigate this timing issue, we consider two alternative specifications. For the first alternative, we assess how our results are affected when we limit the sample to households who report no changes in their food stamp participation status over the past 12 months. Of the original 2702 households, we eliminate 413 households who reported the receipt of food stamps for some months but not others (resulting in a sample size of 2294). For the second alternative, we retain the full sample but shorten the food insecurity and food stamp participation time horizons to the past 30 days. ${ }^{21}$ Frames B and C in Appendix Table 1 display joint frequency distributions analogous to those provided in Table 2.

Table 4 compares critical values obtained from these alternative analyses to those from the primary analysis. Table 4, Row A reproduces critical values from Table 3, Row A (and Figure 4). For example, the sign of $\Delta$ cannot be identified under orthogonal participation errors unless it is known that fewer than $8.2 \%$ of respondents misreport participation ( $2.9 \%$ for the case of food insecurity with hunger). We conservatively focus on the case that food insecurity is assumed to be measured without error. As seen in Table 4 Row B, our main results are fairly insensitive to the exclusion of households reporting part-year food stamp benefits. The self-reported value of $\Delta$ falls from 0.178 to 0.154 , and the critical values of $q$ for identifying the sign of $\Delta$ fall somewhat compared with Row A. In Row $\mathrm{C}$ when the time horizons are shortened to 30 days,

\footnotetext{
${ }^{21}$ In this case, households respond to a shorter list of food insecurity questions. With this shorter list, we can measure food insecurity but not food insecurity with hunger.
} 
the self-reported value $\Delta$ falls to 0.046 . Critical values fall correspondingly under the arbitrary errors and orthogonal errors assumptions. In those cases, the sign of $\Delta$ cannot be identified for participation error rates as low as $2 \%$.

We also assess the sensitivity of the results to other changes in the sample composition. Row D provides critical values for the subsample of single parent households. Row E provides corresponding values for households with incomes below $50 \%$ of the poverty line. Reported food stamp participation rates are higher among these groups (53\% and $63 \%$, respectively) than for our primary sample (41\%). In most cases, conditioning on these characteristics has little effect on self-reported values of $\Delta$ or on the critical values. In any case, we cannot identify the sign of $\Delta$ if food stamp error rates may exceed $10 \%$, even abstracting away from mismeasured food insecurity and uncertainty associated with sampling variability. Collectively, these findings suggest that we should not be confident that food stamp recipient households are less likely to be food secure than nonrecipient households unless we are willing to place a large degree of confidence in the data.

\section{Conclusion}

As the cornerstone of the federal food assistance system, the Food Stamp Program is charged with being the first line of defense against hunger. In this light, researchers and policymakers have been puzzled to observe negative relationships between food security and the receipt of food stamps among observationally similar eligible households. Using a multivariate logit regression framework, for example, Wilde and Nord (2005) estimate a negative impact of food stamp participation on food security even after controlling for unobserved fixed effects. Given no plausible mechanism through which food stamps would diminish food security, they conclude that their estimated coefficient is biased due to unobserved time-varying household characteristics.

While we agree that the paradox might be explained by unobserved characteristics, we also find that the paradox hinges on strong assumptions about the reliability of the data. Food insecurity responses are partially subjective, and evidence from Bollinger and David (1997) and Bitler et al. (2003) suggests that error rates in self-reported food stamp recipiency exceed $12 \%$. We introduced a nonparametric empirical framework for assessing what can be inferred about conditional probabilities when a binary outcome and conditioning variable are both subject to nonclassical measurement error. Even when we impose strong 
assumptions restricting the patterns of classification errors, we find that food stamp participation error rates much smaller than $12 \%$ are sufficient to prevent us from being able to draw firm conclusions about relationships between food stamp participation and food insecurity. The possibility of misreported food insecurity exacerbates the uncertainty.

More generally, our analysis derives easy-to-compute sharp bounds on partially identified conditional probabilities when a binary outcome and a binary conditioning variable are both subject to arbitrary endogenous classification error. The framework can be applied to a wide range of topics in the social sciences involving nonrandom classification errors. We have not, however, attempted to provide a structural model of food stamp eligibility and participation. Our approach, for example, cannot identify the policy impacts of proposed changes in food assistance programs. Instead, our approach is intended to provide a useful starting point for understanding what can be known about relationships between food insecurity and food stamp participation under current policies. We hope that future research aimed at identifying food assistance policy effects will explicitly account for the uncertainty associated with potential reporting errors in the key variables of interest. 


\section{References}

[1] Aigner, D. (1973). "Regression with a Binary Independent Variable subject to Errors of Observations." Journal of Econometrics, 1, 49-60.

[2] Bhattacharya, J., J. Currie, and S. Haider. (2004). "Poverty, Food Insecurity, and Nutritional Outcomes in Children and Adults." Journal of Health Economics, 23(4): 839-62.

[3] Bitler, M., J. Currie, and J.K. Scholz. (2003). "WIC Eligibility and Participation," Journal of Human Resources, 38(S): 1139-1179.

[4] Bitler, M., C. Gundersen, and G. Marquis. (2005). "Are WIC Non-Recipients at Less Nutritional Risk than Recipients? An Application of the Food Security Measure." Review of Agricultural Economics, 27(3): 433-38.

[5] Black, D., S. Sanders, and L. Taylor. (2003). "Measurement of Higher Education in the Census and CPS," Journal of the American Statistical Association, 98 (463), 545-54.

[6] Bollinger, C. (1996). "Bounding Mean Regressions When a Binary Regressor is Mismeasured." Journal of Econometrics, 73: 387-99.

[7] Bollinger, C. and M. David. (1997). "Modeling Discrete Choice with Response Error: Food Stamp Participation." Journal of the American Statistical Association, 92:827-35.

[8] Bollinger, C. and M. David. (2001). "Estimation With Response Error and Nonresponse: Food Stamp Participation in the SIPP." Journal of Business and Economic Statistics, 19: 129-142.

[9] Bollinger, C. and M. David. (2005). "I Didn't Tell, and I Won't Tell: Dynamic Response Error in the SIPP." Journal of Applied Econometrics, 20: 563-569.

[10] Borjas, G. (2004). "Food Insecurity and Public Assistance." Journal of Public Economics, 88: 1421-43.

[11] Bound, J. and Burkhauser, R. (1999). "Economic Analysis of Transfer Programs Targeted on People with Disabilities." In Orley Ashenfelter and David Card (Eds.), Handbook of Labor Economics, Vol. 3C. Amsterdam: Elsevier Science, 3417-3528.

[12] Cunnyngham, K. and B. Brown. (2004). Characteristics of Food Stamp Households: Fiscal Year 2003. U.S. Department of Agriculture, Food and Nutrition Service.

[13] Cunnyngham, K. (2005). Food Stamp Program participation rates: 2003. Washington DC: U.S. Department of Agriculture, Food and Nutrition Service.

[14] Dominitz, J., and R. Sherman. (2004). "Sharp Bounds Under Contaminated or Corrupted Sampling With Verification, With an Application to Environmental Pollutant Data," Journal of Agricultural, Biological and Environmental Statistics, 9(3): 319-38.

[15] Dunifon, R., and L. Kowaleski-Jones. (2003). "The Influences of Participation in the National School Lunch Program and Food Insecurity on Child Well-Being." Social Service Review, 77(1): 72-92.

[16] Efron, B., and R. Tibshirani. (1993). An Introduction to the Bootstrap. London: Chapman and Hall. 
[17] Frazis, H. and M. Loewenstein. (2003). "Estimating Linear Regressions with Mismeasured, Possibly Endogenous, Binary Explanatory Variables," Journal of Econometrics, 117, 151-78.

[18] Furness, B., P. Simon, C. Wold, and J. Asarian-Anderson. (2004). "Prevalence and Predictors of Food Insecurity Among Low-Income Households in Los Angeles County." Public Health Nutrition, 7(6): 791-4.

[19] Gundersen, C. and S. Offutt. (2005). "Farm Poverty and Safety Nets." American Journal of Agricultural Economics, 87(4), 885-99.

[20] Gundersen, C. and V. Oliveira. (2001). "The Food Stamp Program and Food Insufficiency." American Journal of Agricultural Economics, 83(4): 875-87.

[21] Gundersen, C. and D. Ribar. (2005). "Food Insecurity and Insufficiency at Low Levels of Food Expenditures." Institute for the Study of Labor (IZA) Working Paper No. 1594.

[22] Gundersen, C., L. Weinreb, C. Wehler, and D. Hosmer. (2003). "Homelessness and Food Insecurity." Journal of Housing Economics, 12(3): 250-72.

[23] Haider, S., A. Jacknowitz, and R. Schoeni. (2003). "Food Stamps and the Elderly: Why is Participation So Low?" Journal of Human Resources, 38: 1080-1111.

[24] Hamelin, A., M. Beaudry, and J. Habicht. (2002). "Characterization of Household Food Insecurity in Quebec: Food and Feelings" Social Science and Medicine, 54: 119-32.

[25] Hausman, J.A., J. Abrevaya, and F.M. Scott-Morton. (1998). "Misclassification of the Dependent Variable in a Discrete-Response Setting." Journal of Econometrics, 87: 239-69.

[26] Horowitz, J. and C. Manski. (1995). "Identification and Robustness With Contaminated and Corrupted Data." Econometrica 63: 281-302.

[27] Hotz, J., C. Mullins, and S. Sanders. (1997). "Bounding Causal Effects Using Data from a Contaminated Natural Experiment: Analysing the Effects of Teenage Childbearing," Review of Economic Studies, 64(4): 575-603.

[28] Huber, P. (1981), Robust Statistics, New York: Wiley.

[29] Kreider, B. and J. Pepper. (forthcoming a). "Disability and Employment: Reevaluating the Evidence in Light of Reporting Errors." Journal of the American Statistical Association.

[30] ___ and ___ (forthcoming b). "Inferring Disability Status from Corrupt Data." Journal of Applied Econometrics.

[31] Laraia, B., A. Siega-Riz, C. Gundersen, and N. Dole. (2006). "Psychosocial factors and socioeconomic indicators are associated with household food insecurity among pregnant women," Journal of Nutrition, 136: $177-182$.

[32] Manski, C. and J. Pepper. (2000). "Monotone Instrumental Variables: With an Application to the Returns to Schooling," Econometrica, 68(4): 997-1010. 
[33] Marquis, K. and J. Moore. (1990). "Measurement errors in SIPP program reports" in Proceedings of the Bureau of the Census Annual Research Conference, Washington, DC: Bureau of the Census, 721-745.

[34] Moffitt, R. (1983). "An Economic Model of Welfare Stigma." American Economic Review, 73: 1023-35.

[35] Molinari, F. (2005). "Partial Identification of Probability Distributions with Misclassified Data." Working Paper. Department of Economics, Cornell University.

[36] Nord, M., M. Andrews, and S. Carlson. (2004). Household Food Security in the United States, 2003, Washington, DC: U.S. Department of Agriculture, Economic Research Service, Food Assistance and Nutrition Research Report 42.

[37] Pepper, J. (2000). "The Intergenerational Transmission of Welfare Receipt: A Nonparametric Bounds Analysis," Review of Economics and Statistics, 82(3), 472-288.

[38] Ranney, C. and J. Kushman. (1987). "Cash Equivalence, Welfare Stigma, and Food Stamps." Southern Economic Journal, 53: 1011-27.

[39] Ribar, D. and K. Hamrick. (2003). Dynamics of Poverty and Food Sufficiency, Washington, DC: U.S. Department of Agriculture, Economic Research Service, Food Assistance and Nutrition Research Report 33.

[40] Trippe, G. P. Doyle, and A. Asher. (1992). Trends in Food Stamp Program Participation Rates: $19^{76}$ to 1009. Current Perspectives on Food Stamp Participation, Washington DC: U.S. Department of Agriculture, Food and Nutrition Service.

[41] U.S. Department of Agriculture (1999). Food and Nutrition Service. Annual Historical Review: Fiscal year 1997.

[42] Van Hook J., and K. Balistreri. (2006). "Ineligible Parents, Eligible Children: Food Stamps Receipt, Allotments, and Food Insecurity Among Children of Immigrants." Social Science Research, 35, 228-51.

[43] Wilde, P. and M. Nord. (2005). "The Effect of Food Stamps on Food Security: A Panel Data Approach." Review of Agricultural Economics, 27(3): 425-32. 


\section{Appendix A. Upper bound values for Proposition 2}

\section{Case 1:}

$$
U B_{1}=\sup _{\theta_{1}^{-} \in \Theta_{j}^{1}, j=1,2} \frac{p_{11}+\theta_{1}^{-}}{p+\theta_{1}^{-}-\theta_{0 j}^{+}\left(\theta_{1}^{-}\right)}
$$

where $\Theta_{j}^{1} \equiv\left[0, \overline{\theta_{1}^{-}}\right] \cap\left\{\theta_{1}^{-}: \theta_{0 j}^{+}\left(\theta_{1}^{-}\right) \in\left[0, \overline{\theta_{0}^{+}}\right]\right\} \cap\left\{\theta_{1}^{-}: \theta_{1}^{-}+\theta_{0 j}^{+}\left(\theta_{1}^{+}\right) \leq q\right\}$

and $\theta_{0 j}^{+}\left(\theta_{1}^{-}\right) \equiv \frac{p+(-1)^{j} \sqrt{p^{2}-4 \theta_{1}^{-}\left[(1-p)-\theta_{1}^{-}\right]}}{2}$ for $j=1,2$.

\section{Case 2:}

$$
U B_{2}=\sup _{\theta_{0}^{+} \in \Theta_{j}^{2}, j=1,2} \frac{p_{11}+\overline{\theta_{1}^{-}}}{p+\overline{\theta_{1}^{-}}+\theta_{0 j}^{-}\left(\theta_{0}^{+}\right)-\theta_{0}^{+}}
$$

where $\Theta_{j}^{2} \equiv\left[0, \overline{\theta_{0}^{+}}\right] \cap\left\{\theta_{0}^{+}: \theta_{0 j}^{-}\left(\theta_{0}^{+}\right) \in\left(0, \overline{\theta_{0}^{-}}\right]\right\} \cap\left\{\theta_{0}^{+}: \overline{\theta_{1}^{-}}+\theta_{0}^{+}+\theta_{0 j}^{-}\left(\theta_{0}^{+}\right) \leq q\right\}$ and $\theta_{0 j}^{-}\left(\theta_{0}^{+}\right) \equiv \frac{1-p-\overline{\theta_{1}^{-}}+(-1)^{j} \sqrt{(1-p)^{2}-4 \theta_{0}^{+}\left(p-\theta_{0}^{+}\right)}}{2}$ for $j=1,2$.

\section{Case 3:}

$$
U B_{3}=\sup _{\theta_{1}^{+} \in \Theta_{j}^{3}, j=1,2} \frac{p_{11}+\theta_{1 j}^{-}\left(\theta_{1}^{+}\right)-\theta_{1}^{+}}{p+\theta_{1 j}^{-}\left(\theta_{1}^{+}\right)-\theta_{1}^{+}-\overline{\theta_{0}^{+}}}
$$

where $\Theta_{j}^{3} \equiv\left(0, \overline{\theta_{1}^{+}}\right] \cap\left\{\theta_{1}^{+}: \theta_{1 j}^{-}\left(\theta_{1}^{+}\right) \in\left[0, \overline{\theta_{1}^{-}}\right]\right\} \cap\left\{\theta_{1}^{+}: \overline{\theta_{0}^{+}}+\theta_{1 j}^{-}\left(\theta_{1}^{+}\right)+\theta_{1}^{+} \leq q\right\}$ and $\theta_{1 j}^{-}\left(\theta_{1}^{+}\right) \equiv \frac{1-p+(-1)^{j} \sqrt{(1-p)^{2}-4\left(\theta_{1}^{+}+\overline{\theta_{0}^{+}}\right)\left(p-\theta_{1}^{+}-\overline{\theta_{0}^{+}}\right)}}{2}$ for $j=1,2$.

\section{Case 4:}

$$
U B_{4}=\sup _{\theta_{0}^{-} \in \Theta_{j}^{4}, j=1,2} \frac{p_{11}+\overline{\theta_{1}^{-}}-\theta_{1 j}^{+}\left(\theta_{0}^{-}\right)}{p+\overline{\theta_{1}^{-}}+\theta_{0}^{-}-\theta_{1 j}^{+}\left(\theta_{0}^{-}\right)-\overline{\theta_{0}^{+}}}
$$

where $\Theta_{j}^{4} \equiv\left(0, \overline{\theta_{0}^{-}}\right] \cap\left\{\theta_{0}^{-}: \theta_{1 j}^{+}\left(\theta_{0}^{-}\right) \in\left(0, \overline{\theta_{1}^{+}}\right]\right\} \cap\left\{\theta_{0}^{-}: \theta_{1 j}^{+}\left(\theta_{0}^{-}\right)+\overline{\theta_{1}^{-}}+\overline{\theta_{0}^{+}}+\theta_{0}^{-} \leq q\right\}$ and $\theta_{1 j}^{+}\left(\theta_{0}^{-}\right) \equiv \frac{p-2 \overline{\theta_{0}^{+}}+(-1)^{j} \sqrt{p^{2}-4\left(\overline{\theta_{1}^{-}}+\theta_{0}^{-}\right)\left[(1-p)-\left(\overline{\theta_{1}^{-}}+\theta_{0}^{-}\right)\right]}}{2}$ for $j=1,2$. 


\section{Table 1}

Food Insecurity Questions in the Core Food Security Module

Food Insecurity Question

Fraction of Households

Responding Affirmatively

1. Worried food would run out

0.535

2. Food bought did not last

0.426

3. Respondent did not eat balanced meal

0.322

4. Child fed few, low-cost foods

0.399

5. Adult(s) cut/skip meals

0.202

6. Child not fed balanced meals

0.235

7. Respondent ate less than should

0.143

8. Adult(s) skipped meals for 3 or more months

0.203

9. Child not eating enough

0.133

10. Respondent hungry but did not eat

0.099

11. Respondent lost weight

0.055

12. Child's meal size cut

0.033

13. Adult(s) did not eat for whole day

0.038

14. Child was hungry

0.023

15. Adult(s) did not eat for whole day for 3 or more months

0.028

16. Child skipped meal

0.011

17. Child skipped meal(s) for 3 or more months

0.008

18. Child did not eat for whole day

0.002 
Figure 1

Responses to Food Insecurity Questions, by Food Insecurity Status

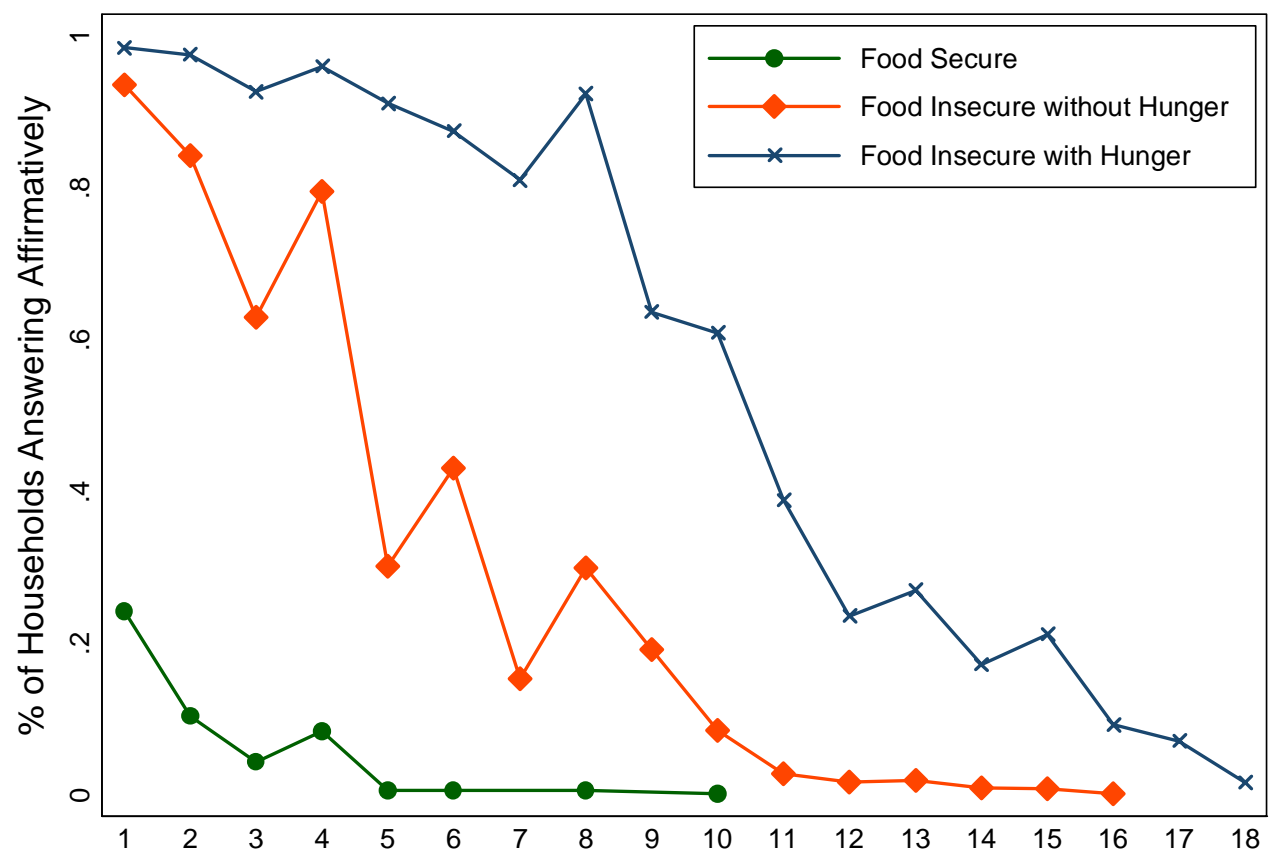

Food Insecurity Question Number 


\section{Table 2}

Reported Food Insecurity Status and Food Stamp Participation Among Eligible Households

\begin{tabular}{|c|c|c|c|}
\hline \multirow{3}{*}{$\begin{array}{l}\text { "Food } \\
\text { Insecure" }\end{array}$} & \multicolumn{2}{|c|}{ A. Food Insecurity } & \multirow{3}{*}{ Totals } \\
\hline & \multicolumn{2}{|c|}{ Food Stamp Participant } & \\
\hline & & & \\
\hline & yes & no & \\
\hline yes & $\begin{array}{c}582 \\
(21.5 \%)\end{array}$ & $\begin{array}{c}549 \\
(20.3 \%)\end{array}$ & $\begin{array}{c}1131 \\
(41.8 \%)\end{array}$ \\
\hline no & $\begin{array}{c}531 \\
(20.3 \%)\end{array}$ & $\begin{array}{c}1045 \\
(38.6 \%)\end{array}$ & $\begin{array}{c}1576 \\
(58.2 \%)\end{array}$ \\
\hline Totals & $\begin{array}{c}1113 \\
(41.1 \%)\end{array}$ & $\begin{array}{c}1594 \\
(58.9 \%)\end{array}$ & $\mathrm{N}=2707$ \\
\hline
\end{tabular}

B. Food Insecurity With Hunger

Food Stamp Participant

"Food Insecure With Hunger"

yes
no
Totals

"'

$$
\text { yes }
$$

177

$(6.5 \%)$

936

$(34.6 \%)$

1113

(41.1\%)

\begin{tabular}{cc} 
& Totals \\
\cline { 2 - 2 } no & \\
& \\
150 & 327 \\
$(5.5 \%)$ & $(12.1 \%)$ \\
& \\
1444 & 2380 \\
$(53.3 \%)$ & $(87.9 \%)$ \\
1594 & $\mathrm{~N}=2707$ \\
$(58.9 \%)$ & \\
\hline
\end{tabular}




\section{Figure 2}

Sharp Bounds on the Prevalence of Food Insecurity Among Households With Children that Receive Food Stamps

Fully Accurate Reporting of Food Insecurity Status,

Potentially Misclassified Food Stamp Recipiency

\section{Food Insecurity Rate Among \\ Food Stamp Recipients}

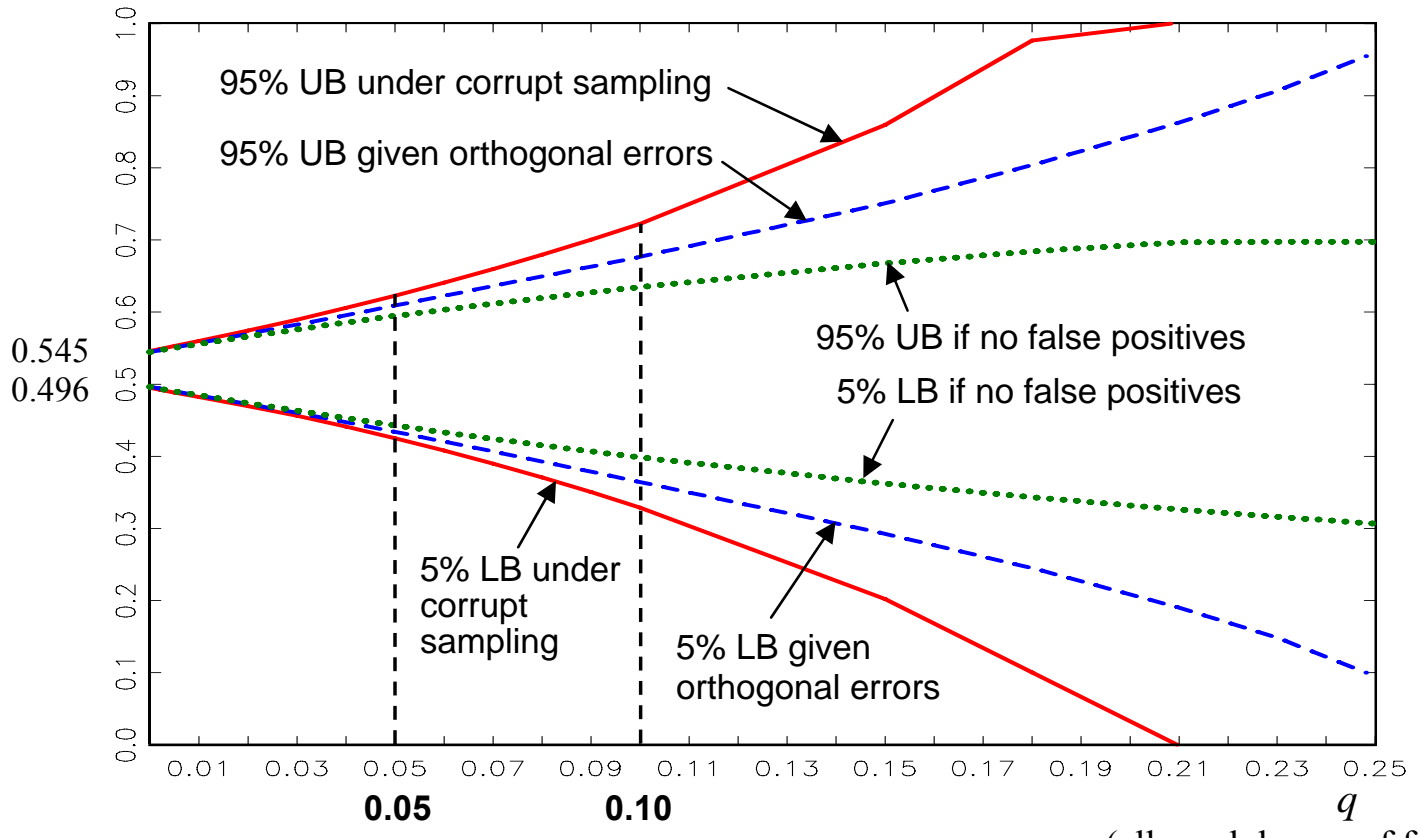

(allowed degree of food stamp participation misclassification)

Corrupt Sampling Orthogonal Errors No False Positive Reports

\begin{tabular}{|c|c|c|c|}
\hline$q=0$ & $\begin{array}{l}{[0.523,0.523]^{\dagger}} \\
{\left[\begin{array}{ll}0.496 & 0.545\end{array}\right]^{\ddagger}}\end{array}$ & $\begin{array}{l}{[0.523,0.523]} \\
{[0.496 \quad 0.545]}\end{array}$ & $\begin{array}{l}{[0.523,0.523]} \\
{[0.496 \quad 0.545]}\end{array}$ \\
\hline$q=0.05$ & $\begin{array}{l}{[0.457,0.595]} \\
{\left[\begin{array}{ll}0.427 & 0.621]\end{array}\right.}\end{array}$ & $\begin{array}{l}{\left[\begin{array}{l}0.461, \\
0.586\end{array}\right]} \\
{\left[\begin{array}{ll}0.434 & 0.609\end{array}\right]}\end{array}$ & $\begin{array}{l}{[0.466,0.575} \\
{\left[\begin{array}{ll}0.443 & 0.595\end{array}\right]}\end{array}$ \\
\hline$q=0.10$ & $\begin{array}{l}{[0.370,0.691]} \\
{\left[\begin{array}{ll}0.330 & 0.721]\end{array}\right.}\end{array}$ & $\begin{array}{l}{[0.396,0.652]} \\
{\left[\begin{array}{ll}0.364 & 0.677\end{array}\right]}\end{array}$ & $\begin{array}{l}{[0.421,0.616]} \\
{[0.3990 .635]}\end{array}$ \\
\hline$q=0.25$ & $\begin{array}{l}{[0.000,1.000]} \\
{[0.0001 .000]}\end{array}$ & $\begin{array}{l}{[0.141,0.916]} \\
{[0.0950 .960]}\end{array}$ & $\begin{array}{l}{[0.325,0.681]} \\
{\left[\begin{array}{ll}0.306 & 0.697\end{array}\right]}\end{array}$ \\
\hline
\end{tabular}

" $5 \% \mathrm{LB} "=5^{\text {th }}$ percentile lower bound; " $95 \%$ UB" $=95^{\text {th }}$ percentile upper bound

${ }^{\dagger}$ Point estimates of the population bounds

${ }^{\ddagger}$ Bootstrapped $5^{\text {th }}$ and $95^{\text {th }}$ percentile bounds (1,000 pseudosamples) 


\section{Figure 3}

Sharp Bounds on the Prevalence of Food Insecurity Among Eligible Households that Do Not Receive Food Stamps

Fully Accurate Reporting of Food Insecurity Status, Potentially Misclassified Food Stamp Recipiency

\section{Food Insecurity Rate Among \\ Food Stamp Nonrecipients}

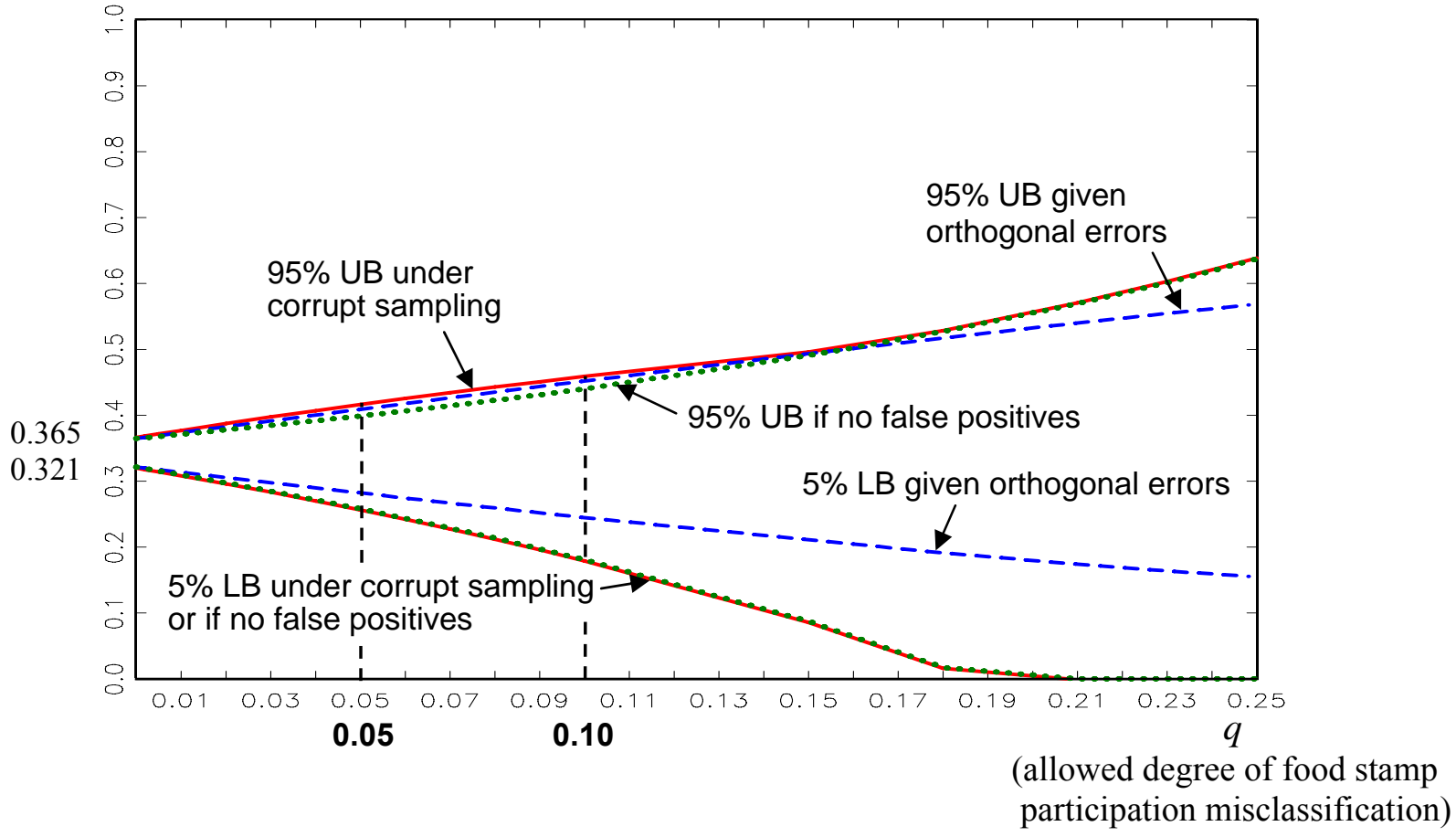

Corrupt Sampling Orthogonal Errors No False Positive Reports

\begin{tabular}{|c|c|c|c|}
\hline$q=0$ & {$[0.344,0.344]^{\dagger}$} & {$[0.344,0.344]$} & {$[0.344,0.344]$} \\
\hline & {$\left[\begin{array}{ll}0.321 & 0.365\end{array}\right]^{\ddagger}$} & {$\left[\begin{array}{ll}0.321 & 0.365\end{array}\right]$} & {$\left[\begin{array}{ll}0.321 & 0.365\end{array}\right]$} \\
\hline$q=0.05$ & {$[0.284,0.396]$} & {$[0.305,0.389]$} & {$[0.284,0.376]$} \\
\hline & {$\left[\begin{array}{ll}0.258 & 0.415\end{array}\right]$} & {$\left[\begin{array}{ll}0.282 & 0.409\end{array}\right]$} & {$\left[\begin{array}{ll}0.258 & 0.399\end{array}\right]$} \\
\hline$q=0.10$ & {$[0.210,0.440]$} & {$[0.268,0.432]$} & {$[0.210,0.415]$} \\
\hline & {$\left[\begin{array}{ll}0.181 & 0.458\end{array}\right]$} & {$\left[\begin{array}{ll}0.245 & 0.452\end{array}\right]$} & {$\left[\begin{array}{ll}0.181 & 0.440\end{array}\right]$} \\
\hline$q=0.25$ & {$[0.000,0.599]$} & {$[0.181,0.549]$} & {$[0.000,0.599]$} \\
\hline & {$\left[\begin{array}{ll}0.000 & 0.637\end{array}\right]$} & {$\left[\begin{array}{ll}0.155 & 0.568\end{array}\right]$} & {$\left[\begin{array}{ll}0.000 & 0.637\end{array}\right.$} \\
\hline
\end{tabular}

" $5 \% \mathrm{LB} "=5^{\text {th }}$ percentile lower bound; " $95 \% \mathrm{UB} "=95^{\text {th }}$ percentile upper bound

${ }^{\dagger}$ Point estimates of the population bounds

${ }^{\ddagger}$ Bootstrapped $5^{\text {th }}$ and $95^{\text {th }}$ percentile bounds (1,000 pseudosamples) 


\section{Figure 4}

Sharp Bounds on the Difference in Food Insecurity Prevalence Rates Between Food Stamp Recipients and Nonrecipients (Among Eligible Households with Children)

Fully Accurate Reporting of Food Insecurity Status,

Potentially Misclassified Food Stamp Recipiency

\section{Difference in Food Insecurity}

Prevalence Rates $(\Delta)$

\section{A. Food Insecurity}

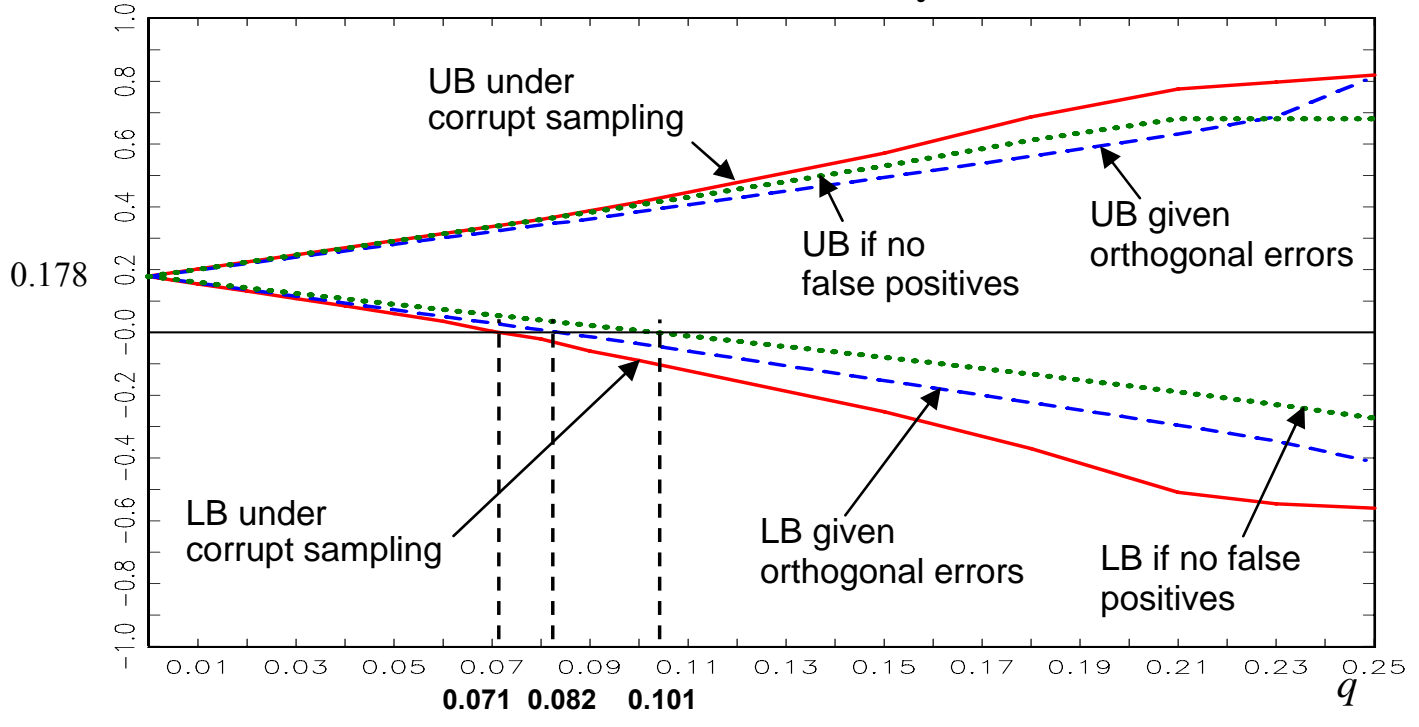

\section{B. Food Insecurity with Hunger}

Difference in Food Insecurity With

Hunger Prevalence Rates $(\Delta)$

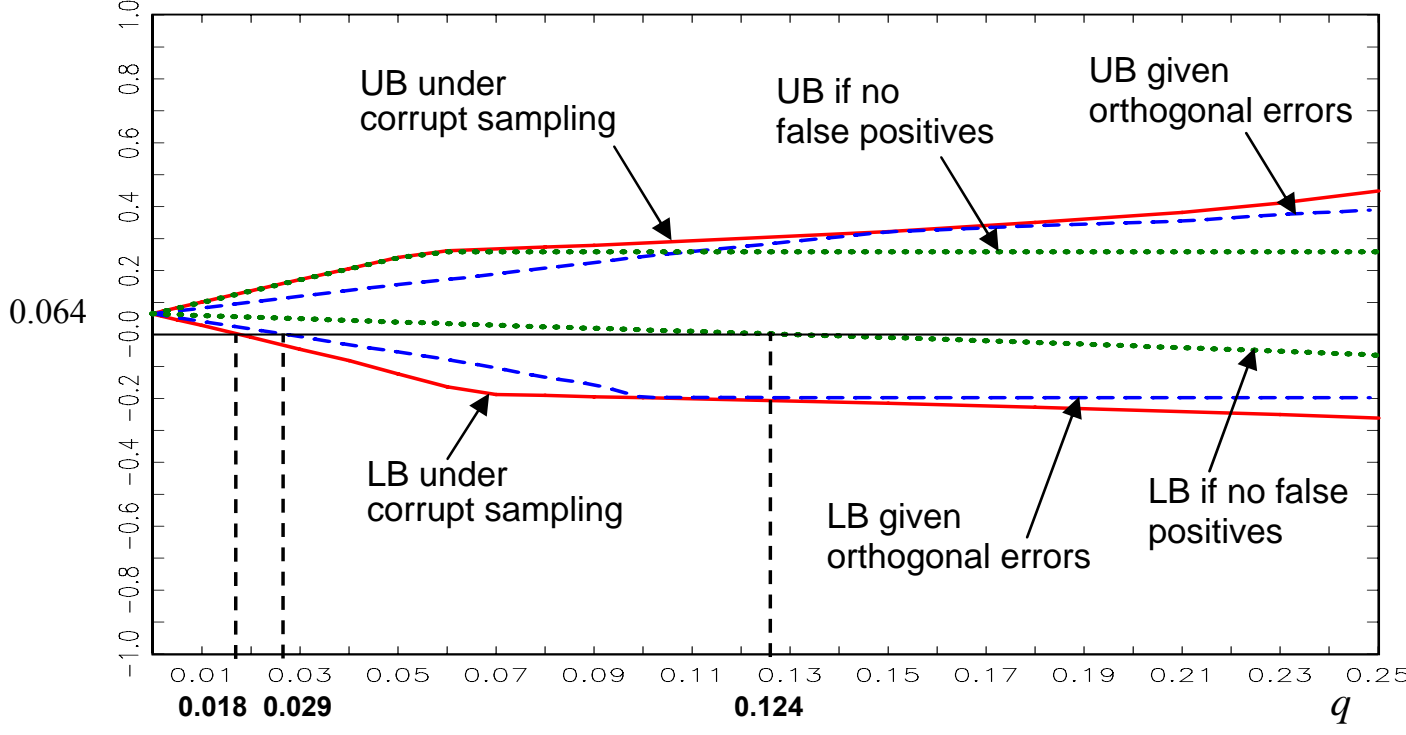

\footnotetext{
${ }^{\dagger}$ Point estimates of the population bounds
} 


\section{Table 3}

Critical Values for the Maximum Allowed Degree of Food Stamp Recipiency Misclassification Before the Sign of the Food Insecurity Gap, $\Delta$, is No Longer Identified

Type of Classification Error in Food Stamp Participation Status, $X^{*}$

Type of Classification Error

in Food Insecurity Status, $Y^{*}$
A. No food insecurity errors

B. Orthogonal errors: $P\left(Y^{*}=1 \mid Z^{\prime}\right)=P\left(Y^{*}=1\right)$ with $q^{\prime}=0.05^{\mathbf{b}}$

C. Arbitrary errors (corrupt sampling) with $q^{\prime}=0.05^{\mathrm{c}}$ (i) Arbitrary errors (corrupt sampling) (ii) Orthogonal errors: $P\left(X^{*}=1 \mid Z^{\prime}\right)=P\left(X^{*}=1\right)$ (iii) No False Positive Classifications

\section{Critical value of $q:^{\mathrm{a}}$}

0.071

0.028

0.021
Critical value of $q$ :

0.082

0.033

0.024
Critical value of $q$ :

0.101

0.041

0.035

${ }^{a}$ Maximum allowed degree of reporting error in food stamp participation, $q^{\mathrm{c}}$, such that the sign of $\Delta$ is no longer identified for

higher allowed error rates. Critical values are conservatively large in that they do not account for sampling variability.

' If $q$ ' exceeds 0.084 , the sign of $\Delta$ is not identified even if all food stamp participation responses are known to be accurate (i.e., $q=0$ ).

'If $q$ ' exceeds 0.073 , the sign of $\Delta$ is not identified even if all food stamp participation responses are known to be accurate (i.e., $q=0$ ). 


\section{Table 4}

Critical Values for the Maximum Allowed Degree of Food Stamp Recipiency Misclassification Before the Sign of the Food Insecurity Gap, $\Delta$, is No Longer Identified: Alternative Time Frames and Samples

Type of Classification Error in Food Stamp Participation Status, $X^{*}$
(i) Food Insecurity
(ii) Food Insecurity With Hunger

\section{$\underline{\text { Self-reported } \Delta}$}
(i) 0.178
(ii) 0.065

(i) Arbitrary errors

(corrupt sampling)

(ii) Orthogonal errors:

$P\left(X^{*}=1 \mid Z^{\prime}\right)=P\left(X^{*}=1\right)$

(iii) No False Positive

(corrupt sampling)

\section{Critical value of $q:^{\dagger}$}
0.071
0.018
0.053
0.011

$\begin{array}{ll}\text { (i) } & 0.154 \\ \text { (ii) } & 0.049\end{array}$

Receipt for all 12 Months or

Zero Months $(\mathrm{N}=2294)$
C. Food Stamp Participation and
Food Insecurity Defined over
Previous 30 Days $(\mathrm{N}=2707)$

\section{Single Parent Households} $(\mathrm{N}=1514)$

\section{(i) 0.046 \\ (ii) $\mathrm{NA}^{*}$}
0.012
NA

(i) 0.138

(ii) 0.051

(i) 0.187

(ii) 0.064
$50 \%$ of Poverty Line $(\mathrm{N}=696)$

\section{Critical value of $q$ :}

0.082

0.029

0.060

0.015

0.017

NA

0.066

NA

$\begin{array}{lll}0.064 & 0.070 & 0.076 \\ 0.014 & 0.027 & 0.088 \\ & & \\ 0.075 & 0.090 & 0.098 \\ 0.066 & 0.073 & 0.080\end{array}$

${ }^{\dagger}$ Maximum allowed degree of reporting error in food stamp participation, $q^{\mathrm{c}}$, such that the sign of $\Delta$ is no longer identified for higher allowed error rates. Critical values are conservatively large in that (1) they do not account for sampling variability and (2) food insecurity is assumed to be measured without error. ${ }^{+}$In this case, the data are insufficient to measure food insecurity with hunger. 


\section{Appendix Table 1}

Reported Food Insecurity Status and Food Stamp Participation Among Eligible Households:

Alternative Time Frames and Samples

A. Base values (see Table 1, $\mathrm{N}=\mathbf{2 2 9 4}$ )

B. Households Reporting Food Stamp Receipt for all 12 Months or Zero Months (N=2294)

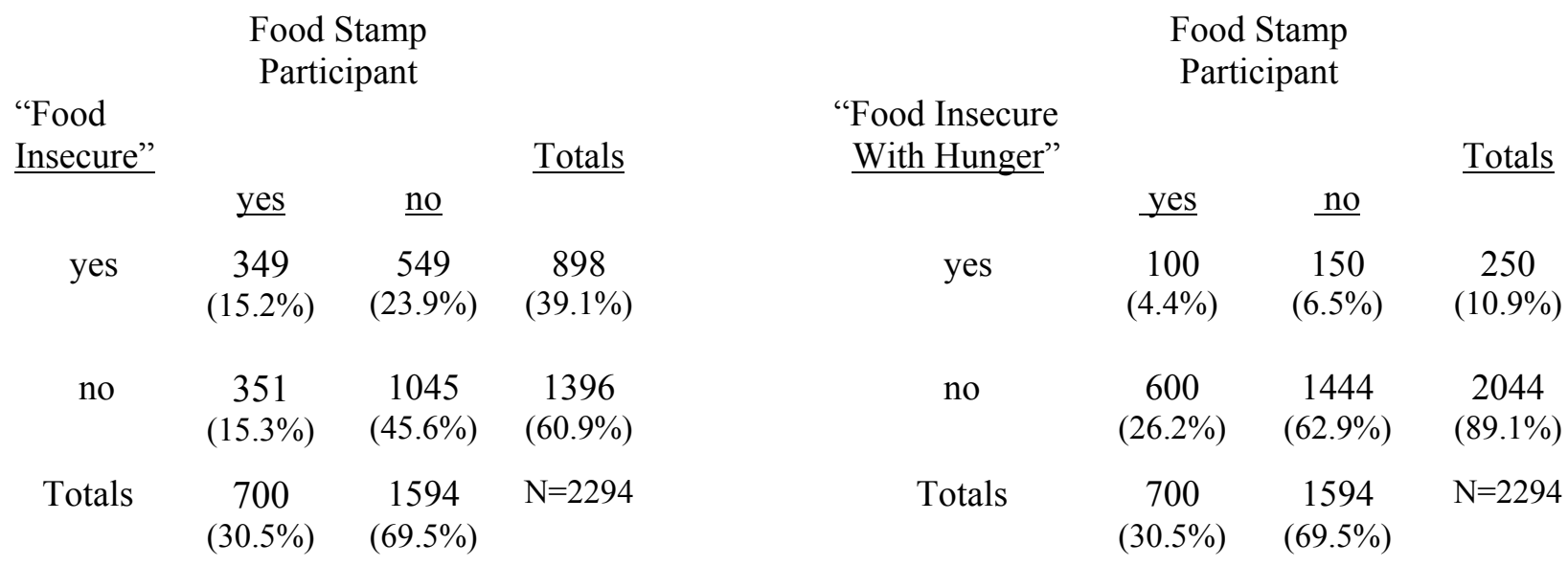

C. 30-Day Measures ( $\mathrm{N}=\mathbf{2 7 0 7})$

\begin{tabular}{|c|c|c|c|c|c|c|c|}
\hline \multirow{2}{*}{$\begin{array}{l}\text { "Food } \\
\text { Insecure" }\end{array}$} & \multicolumn{2}{|c|}{$\begin{array}{c}\text { Food Stamp } \\
\text { Participant }\end{array}$} & & & \multicolumn{2}{|c|}{$\begin{array}{c}\text { Food Stamp } \\
\text { Participant }\end{array}$} & \\
\hline & & & & "Food Insecure & & & \\
\hline & yes & $\underline{\text { no }}$ & & & yes & no & \\
\hline yes & $\begin{array}{c}178 \\
(6.6 \%)\end{array}$ & $\begin{array}{c}251 \\
(9.3 \%)\end{array}$ & $\begin{array}{c}429 \\
(15.8 \%)\end{array}$ & yes & NA & NA & NA \\
\hline no & $\begin{array}{c}767 \\
(28.3 \%)\end{array}$ & $\begin{array}{c}1511 \\
(55.8 \%)\end{array}$ & $\begin{array}{c}2278 \\
(84.2 \%)\end{array}$ & no & NA & NA & NA \\
\hline Totals & $\begin{array}{c}945 \\
(34.9 \%)\end{array}$ & $\begin{array}{c}1762 \\
(65.1 \%)\end{array}$ & $\mathrm{N}=2707$ & Totals & $\begin{array}{c}945 \\
(34.9 \%)\end{array}$ & $\begin{array}{c}1762 \\
(65.1 \%)\end{array}$ & $\mathrm{N}=2707$ \\
\hline
\end{tabular}

$\mathrm{NA}=$ not available 


\section{Single Parent Households $(\mathrm{N}=1514)$}

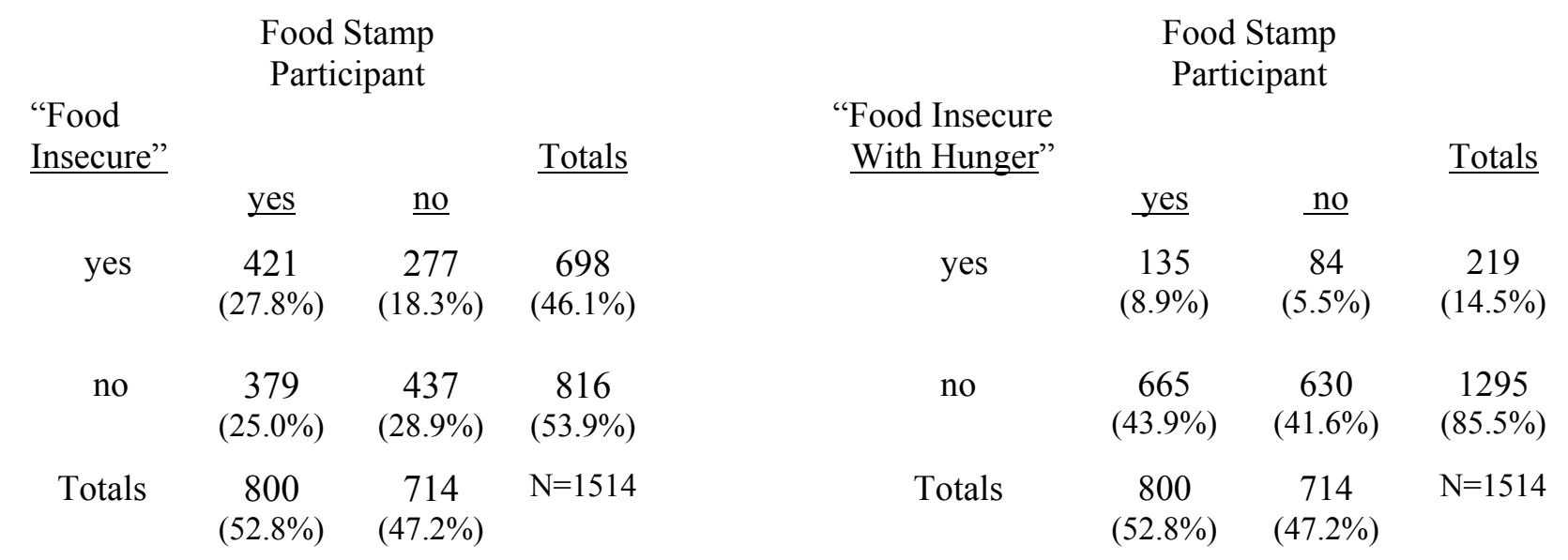

E. Households with Incomes Below $50 \%$ of Poverty Line $(\mathbf{N}=696)$

\section{Food Stamp}

Participant

"Food

Insecure"

$\begin{array}{cccc} & \text { yes } & \text { no } & \\ \text { yes } & 226 & 83 & 309 \\ & (27.8 \%) & (18.3 \%) & (44.4 \%) \\ \text { no } & 215 & 172 & 387 \\ & (25.0 \%) & (28.9 \%) & (55.6 \%)\end{array}$

Totals

$441 \quad 255 \quad \mathrm{~N}=696$

Food Stamp

Participant

"Food Insecure

With Hunger"

$\underline{\text { Totals }}$

$\begin{array}{cccc} & \text { yes } & \text { no } & \\ \text { yes } & 68 & 23 & 91 \\ & (9.8 \%) & (3.3 \%) & (13.1 \%)\end{array}$

no

$373 \quad 232 \quad 605$

$(53.6 \%) \quad(33.3 \%) \quad(86.9 \%)$

Totals

\begin{tabular}{ccc}
441 & 255 & $\mathrm{~N}=696$ \\
$(63.4 \%)$ & $(36.6 \%)$ & \\
\hline
\end{tabular}

